\title{
Movement Disorders Due to Selective Basal Ganglia Lesions with Uremia
}

\author{
Sherifa Hamed (D, Khaled Mohamed, Sameer Abd Elhameed, Ehab Moussa, \\ Hossam Abozaid, Anthony Lang, Amany Mohamed, Jacklin Moussa
}

\begin{abstract}
Background: Basal ganglia (BG) lesions are rarely reported in patients with uremia and may manifest by movement disorders. However, their exact incidence and pathogenesis have not been extensively studied. This study aimed to determine the frequency, types, risk variables (clinical, laboratory, and imaging), and manifestations of BG lesions with uremia and patients' neurologic outcomes. Methods: This observational study included 70 adults (mean age: $45.87 \pm 3.36$ years; duration of uremia: $5.5 \pm 1.5$ years). They underwent extensive evaluations (clinical, laboratory, and neuroimaging) and had prospectively evaluated clinically every 3 months for 2 years. Repeated magnetic resonance imaging (MRI) brains were done to patients with movement disorders and correlated with their neurologic outcomes. Results: BG lesions were found in 15 patients (21.4\%) and 6 (8.6\%) had movement disorders [Parkinsonism $(n=4)$, choreo-dystonia $(n=1)$ and dystonia $(n=1)]$ after the onset of uremia (mean $=10$ months). There were no characteristic risk variables that distinguished patients with movement disorders from those without. Five developed movement disorders prior to the period of the study and one was de novo. The majority was females and had diabetes and higher frequencies of abnormal renal dysfunction, metabolic derangements, and white matter hyperintensities in MRIs. Movement disorders persisted in all patients despite the resolution of neuroimaging in three patients. Conclusions: There is no clear threshold for renal failure to result in movement disorders due to BG lesions. The clinical outcome is variables depending on each patient's comorbidities and complications. Persistent neuronal damage (due to uremic toxins/metabolic/nutritional and ischemic/microvascular factors) has been suggested as the cause of poor neurologic outcomes.
\end{abstract}

RÉSUMÉ : Des troubles du mouvement attribuables à des lésions sélectives des ganglions de la base chez des patients urémiques. Contexte : Rarement signalées chez des patients urémiques, des lésions aux ganglions de la base peuvent se traduire par des troubles du mouvement. Cela dit, leur incidence exacte, de même que leur pathogénèse, n'ont pas encore fait l'objet d'études approfondies. Cette étude entend donc déterminer la fréquence et les types de lésions des ganglions de la base ainsi que leurs variables de risque (sur le plan clinique, en laboratoire et par IRM), leurs manifestations lorsqu'associées à l'urémie et l'évolution de l'état neurologique des patients qui en sont atteints. Méthodes : Cette étude observationnelle a inclus 70 adultes (âge moyen : 45,87 \pm 3,36 ans ; durée de l'urémie : 5,5 $\pm 1,5$ ans). Ils ont dû subir des évaluations approfondies (de type clinique, en laboratoire et par neuro-imagerie) et ont été ensuite évalués sur le plan clinique à tous les 3 mois pendant 2 ans. Des examens d'IRM ont également été effectués de façon répétée chez les patients atteints de troubles du mouvement. Leurs résultats ont été par la suite corrélés avec l'évolution de leur état neurologique. Résultats : On a trouvé des lésions aux ganglions de la base chez 15 patients, soit 21,4\% d'entre eux ; sur ces 15 patients, 6, soit 8,6\% du nombre total, étaient atteints de troubles du mouvement [parkinsonisme $(n=4)$, chorée-dystonie $(n=1)$ et dystonie $(n=1)$ ] après l'apparition de symptômes urémiques (moyenne $=10$ mois). Aucune variable de risque caractéristique permettant de distinguer les patients avec ou sans troubles du mouvement n'a été détectée. Sur ces 6 patients, mentionnons que 5 d'entre eux avaient commencé à développer des troubles du mouvement avant que ne débute notre étude. La majorité d'entre eux était de sexe féminin, souffrait de diabète et montrait des fréquences plus élevées de dysfonction rénale, de dérèglement métabolique et d'hyper-intensité de la substance blanche lors d'examens d'IRM. Enfin, des troubles du mouvement ont persisté chez tous les patients en dépit de la résolution de la neuro-imagerie chez trois patients. Conclusion : Il n'existe pas de seuil clair d'insuffisance rénale pouvant résulter en troubles du mouvement attribuables à des lésions des ganglions de la base. L'évolution de l'état de santé des patients sur le plan clinique variera selon les comorbidités et les complications de chacun d'entre eux. Nous sommes ainsi amenés à penser que la cause de la faible amélioration de l'état neurologique des patients pourrait être des dommages neuronaux persistants attribuables à des toxines urémiques et à une série de facteurs métaboliques, nutritionnels, ischémiques et microvasculaires.

Keywords: Uremia, Chronic kidney disease, Basal ganglia lesions, Basal ganglia-related movement disorders

doi:10.1017/cjn.2020.29

Can J Neurol Sci. 2020; 47: 350-365

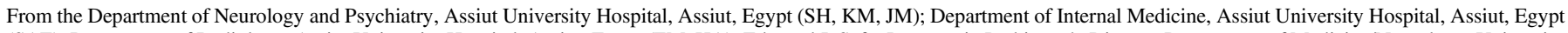

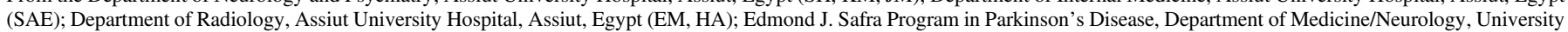
Health Network, University of Toronto, Toronto, Canada (AL); Department of Biochemistry, Assiut University Hospital, Assiut, Egypt (AM)

Received August 8, 2019. Final Revisions Submitted February 3, 2020. Date of Acceptance February 5, 2020.

Correspondence to: Sherifa Hamed, Department of Neurology and Psychiatry, Assiut University Hospital, P.O. Box 71516, Assiut, Egypt. Email: hamed_sherifa@yahoo.com 


\section{INTRODUCTION}

Movement disorders are one of the complications of uremia due to chronic kidney disease (CKD). The common movement disorders with uremia include restless leg syndrome (RLS), ${ }^{1}$ asterixis, ${ }^{2}$ and myoclonus. ${ }^{3}$ Movement disorders, generally believed to be due to basal ganglia (BG) dysfunction (often referred to as "extrapyramidal"), have been rarely reported in patients with uremia. The exact incidence of movement disorders with uremia is unknown. The majority of reported cases with movement disorders had severe uremia and they were most often due to diabetic nephropathy. They included Parkinsonism, ${ }^{4-13}$ chorea, ${ }^{14-22}$ and dystonia. ${ }^{23}$ Acute reversible movement disorders due to bilateral BG lesions had been emphasized in patients with uremia. However, the neurologic outcomes of patients were quite variable. Some reported chronic or permanent movement disorders despite the reversibility or recovery of neuroimaging manifestations.

The BG lesions with uremia have been suggested to be due to an increased susceptibility of BG to injury from uremia toxins and its associated metabolic derangements as metabolic acidosis, lactic acidosis, etc. ${ }^{8}$ particularly in the presence of underlying comorbid vessel disease such as diabetes, arteriosclerosis, atherosclerosis or vasculitis, ${ }^{4-6,9,10,17,19}$ demyelination, ${ }^{24}$ accumulation of calcium in BG (secondary to hyperparathyroidism), ${ }^{25,26}$ accumulation of manganese in $\mathrm{BG},{ }^{7,11}$ and accelerated loss of thiamine after hemodialysis or due to malnutrition. ${ }^{4,15}$

This was a longitudinal observational study done on a cohort of patients with uremia due to CKD. The aims of this study were to determine: (1) the frequency and types of BG lesions and their related movement disorders in patients with uremia, (2) whether patients with BG lesions had different demographic, clinical, laboratory, and neuroimaging findings (risk variables) which distinguished them from the entire studied population or from patients without BG lesions, (3) whether patients with movement disorders had different risk variables (clinical, laboratory, and imaging) which distinguished them from patients without clinical manifestations, and (4) the outcomes (clinical and neuroimaging) of patients with BG-related movement disorders.

\section{Methods}

\section{Participants}

This was a longitudinal prospective observational study carried out over a period of 2 years (July 2016-June 2018). It included 70 patients (age range: $18-62$ years) with chronic renal failure (or uremia due to $\mathrm{CKD}$ ). Patients were recruited consecutively from the Department of Internal Medicine of Assiut University Hospital (a tertiary referral center), Assiut, Egypt, and its out-patient clinic and dialysis unit.

Inclusion criteria were: (1) adults with uremia due to renal cause, (2) cooperation during neurologic evaluation and completion of follow-ups (till the end of study period). Exclusion criteria were: (1) patients with uremia due to pre-renal or post-renal causes, (2) patients with movement disorders prior to the onset of uremia, (3) BG lesion due to other fulminant medical disease (e.g. hepatic failure, HIV, etc.), (4) intake of drugs which are incriminated in movement disorders [e.g. metoclopramide, calcium channel blockers (as diltiazem, nifedipine, and verapamil), monoamine depleters (reserpine and tetrabenazine), antiarrhythmic drugs (as amiodarone), some anticonvulsants (as phenytoin, sodium valproate, etc.)], (5) alcoholism, (6) total parenteral nutrition or regular intake of thiamine supplements before participation in the study, and (7) contraindication for magnetic resonance imaging (MRI). This study also included 30 healthy subjects which were matched for age (range: $22-60$ years, $42.5 \pm 2.4$ years) and sex $($ male $=15$; female $=15)$ recruited from the general population as controls for statistical comparisons of laboratory data. The protocol of the study was done in accordance with the ethical standards of the national research committee and with Helsinki declaration and its later amendments or comparable ethical standards. Informed consent was obtained from all participants.

\section{Methods}

All underwent: (1) detailed medical and neurological histories and examinations. Interviewing of the patients who experienced uremic encephalopathy was repeated when they were not encephalopathic. (2) Laboratory panel for assessment of uremia, (3) Electroencephalography (EEG), (4) Conventional MRI of the brain. For comparisons, patients were categorized into two groups according to the presence or absence of selective BG lesions and their related movement disorders, and (5) Measurement of total blood thiamine [(Vitamin B1 (thiamine) kits, ABC Diagnostic Egypt)] and venous lactate (Spectrum Diagnostics Liquizyme Lactate kit; Egyptian Company of Biotechnology S.A.E)] levels. (6) Regular clinical neurologic evaluation every 3 months till the end of the study ( 2 years). Patients who developed BG-related movement disorders after the onset of uremia $(n=6)$ underwent repeated neuroimaging of the brain (discussed below). For patients who developed movement disorders before the period of the study $(n=5)$, we also reviewed their detailed clinical manifestations and previous laboratory results and neuroimaging.

\section{Statistical Analysis}

Data were analyzed using SPSS version 16.0 for windows (Statistical Package for the Social Sciences Inc, Chicago, IL, USA). Data were expressed as mean (standard deviation or SD). Categorical variables were analyzed using $\chi^{2}$ text and a one-tailed Chi-square analysis. Continuous data were compared using unpaired Student's $t$-tests (two-tailed).

\section{Results}

This study included a total of 70 adults (male $=33$; female $=37$ ) with uremia due to CKD. They had mean age of $45.8 \pm 3.4$ years and duration of uremia of $5.5 \pm 1.5$ years. Forty-five patients $(64.3 \%)$ were on regular hemodialysis (range: 1-13 years; $3.1 \pm 1.6$ years) due to advanced stage of kidney failure (stage 4-5; glomerular filtration rate $[\mathrm{GFR}]<30$ to $<15 \mathrm{ml} / \mathrm{min}$ ). The number of dialysis sessions varied from one to three sessions per week (mean: $3 \pm 1$ ) with the mean session duration of $3.5 \pm 0.5 \mathrm{~h}$ (range: $2.5-4 \mathrm{~h})$. The remaining $(n=25,35.7 \%)$ had stage 3 kidney failure (GFR: $30-59 \mathrm{ml} / \mathrm{min}$ ) and were on dietary control and supportive treatments. The common causes of uremia were nephritis (64.3\%) and diabetic nephropathy (24.3\%). Hypertension and ischemic heart disease were the commonest comorbid medical conditions $(70 \%)$. History of development of recurrent seizures (epilepsy) after the onset of uremia was reported in $15.7 \%(n=11$; on dialysis $=6$, not on dialysis $=5$ ). Epileptic EEG activity was reported in $24.3 \%(n=17)$ in absence of history of epilepsy 
(subclinical). Uremic encephalopathy was reported in $45.7 \%$ ( $n$ =32). All patients with uremic encephalopathy were on regular dialysis. Peripheral neuropathy was reported in 67\% $(n=47)$.

The frequent abnormal involuntary movements observed in patients with uremia were asterixis $(n=42 ; 60 \%)$, myoclonus $(n=48 ; 68.6 \%)$, and RLS $(n=38 ; 53.3 \%)$. Asterixis and myoclonus were present in every patient with uremic encephalopathy. Myoclonic jerking was observed in $40 \%(n=10)$ of patients not on dialysis. EEG of the majority of patients with myoclonus (67\%) showed spikes or polyspikes and slow wave activities which had temporal relationship to the muscle action potentials associated with the myoclonic jerks. RLS was present in patients with uremia regardless to the degree of kidney failure, presence or absence of peripheral neuropathy or anemia. Muscle cramps were reported in $41.4 \%$ of patients $(n=29)$ particularly with hypocalcemia. Tetany was found in six patients $(8.6 \%)$ with calcium levels less than $6 \mathrm{mg} / \mathrm{dl}(1.5 \mathrm{mmol} / \mathrm{l})$. Four patients with tetany also had generalized tonic-clonic seizures. BG-related movement disorders were found in six patients $(8.6 \%)$ (Parkinsonism $=4$; choreo-dystonia $=1$; dystonia $=1)($ Table 1$)$.

The most frequent laboratory abnormalities observed in studied patients were hypocalcemia $(70 \%)$, low red blood cells (RBCs) count and anemia (68.6\%), and low thiamine concentrations $(68.6 \%)$. None of the patients had lactic acidosis or thiamine deficiency (defined as thiamine concentrations less than $9 \mathrm{nmol} / \mathrm{l})$. Twenty-nine patients with low thiamine concentrations also had low albumin levels $(60.4 \%$ or $29 / 48)$ (Supplementary Table 1).

The most frequent MRI-brain abnormalities observed in patients with uremia were white matter hyperintensities (WMHs) (48.6\%, $n=34$ ) which defined as small periventricular and subcortical hyperintense lesions in T2-weighted image (T2WI) and fluid attenuated inversion recovery (FLAIR). Focal encephalomalacia in cerebral hemispheres (lobar) (iso-signal to CSF) was observed in $18.6 \%$. In absence of clinical stroke manifestation, these lesions might be a consequence of silent brain infarcts. BG lesions were found in $21.4 \%(n=15)$ (Table 1). Bilateral reversible BG lesions were found in $4.3 \%(n=3)$ (discussed below). Bilateral BG calcifications were found in $10 \%(n=7)$. Unilateral BG focal encephalomalacia was found in $7 \%(n=5)$.

Compared to the entire studied population and patients without BG lesions, the majority of patients with $\mathrm{BG}$ lesions were females $(P=0.01)$ and had diabetic nephropathy $(P=0.0001)$, history of uremic encephalopathy $(P=0.01)$, RLS $(P=0.01$ and $P=0.001$, respectively), higher median levels creatinine ( $P=0.01$ and $P=0.001$, respectively), hyponatremia $(P=0.01$ and $P=0.001$, respectively), hypocalcemia $(P=0.01$ and $P=0.001$, respectively), hyperphosphatemia $(P=0.03$ and $P=0.001$, respectively), anemia $(P=0.001)$, and WMHs in their MRI-brains ( $P=0.03$ and $P=0.02$, respectively). Four patients with BG calcifications had tetany and hyperphosphatemia and two had elevated parathyroid hormone levels. No significance differences had been identified between the three groups in relation to age, age at onset of uremia, duration of uremia or whether being on dialysis or not, presence or absence of metabolic acidosis, and thiamine concentrations. Six patients with BG lesions had extrapyramidal movement disorders. If we excluded the seven patients with BG calcification, therefore $75 \%(6 / 8)$ of patients with BG lesions had extrapyramidal movement disorders. They developed uremia at ages ranged between 50 and
58 years $($ median $=55$ years $)$. Four $(66.7 \%)$ were females. Diabetic nephropathy was the cause of uremia in four patients $(66.7 \%)$ and two $(33.3 \%)$ patients had comorbid hypertension. Patients developed movement disorders after the onset of uremia by $3-24$ months (median $=10$ months). The details of the case series of patients with BG-related movement disorders are demonstrated below and summarized in Table 2 .

\section{Case series of patients with BG-related movement disorders}

\section{Case 1}

A 58-year-old female had a 10-year history of type 2 diabetes (T2D) causing diabetic nephropathy and uremia (stage 4; GFR: $23 \mathrm{ml} / \mathrm{min}$ ) at the age of 55 . She had been on regular hemodialysis for a year (three sessions per week, $3 \mathrm{~h}$ each), metformin $(500 \mathrm{mg}$ / bid), insulin (30 U/d), vitamin B complex, and calcium supplement tablets. Her detailed history revealed that she developed movement disorder after the onset of uremia by 1 year (i.e. at the age of 56). She described development of acute onset of tremors in her hands (left > right) which occurred during rest and intensified with action particularly with posture maintenance. She had MRI-brain within the first week after the onset of the neurologic condition which showed lesions in the right lentiform nucleus, left putamen, and the right occipital lobe. These lesions were hypointense in T1-weighted image (T1WI) and hyperintense in T2WI, fluid attenuated inversion recovery (FLAIR), and diffusion-weighted image (DWI). The ventricles appeared normal and there was no evidence of mass effect (Figure 1 (A-D)). Her laboratory investigations were significant for higher urea and creatinine than her usual, hyperglycemia, metabolic acidosis, anemia, and mild hypocalcemia [urea: $15 \mathrm{mmol} / \mathrm{l}$; creatinine: $1100 \mu \mathrm{mol} / \mathrm{l} ;$ random blood sugar (RBS): $15 \mathrm{mmol} / \mathrm{l}$; calcium: $2.05 \mathrm{mmol} / \mathrm{l}(8.2 \mathrm{mg} / \mathrm{dl})$; sodium: $133 \mathrm{mmol} / \mathrm{l}$; potassium: $3.5 \mathrm{mmol} / \mathrm{l}$; phosphorus: $3.8 \mathrm{mg} / \mathrm{dl}$; magnesium: $2.3 \mathrm{mg} / \mathrm{dl}$; hemoglobin (HGB): $6 \mathrm{mg} / \mathrm{dl}$; RBCs count: $2.6 \mathrm{million} / \mathrm{mcl}$; $\mathrm{PH}$ : 7.30; PO2: 95 mmHg; PCO2: $40 \mathrm{mmHg}$; HCO3: $11 \mathrm{MEq} / \mathrm{l}]$. She denied history of uremic encephalopathy. Neurological consultation (at the age of 56) confirmed the diagnosis of Parkinsonian rest tremors. The patient refused to receive treatment for her tremors. Follow-up MRI-brain (done 1 month after the onset of the neurologic condition) was unremarkable despite the persistence of tremors. Neurological evaluation (at the age of 58, first interviewing during the study period) showed an alert and cooperative female. She had sensory peripheral neuropathy in her lower limbs, RLS, and frequent muscle cramps (1-2 times/ week). She denied deterioration of her neurologic condition except worsening of her tremors in the day before dialysis. Her blood pressure was $110 / 70 \mathrm{mmHg}$, heart rate was 100 beats $/ \mathrm{min}$, respiratory rate was 26 breaths $/ \mathrm{min}$, and temperature was $36.0^{\circ} \mathrm{C}$. She had no evidence of cranial nerves or cerebellar abnormalities. Neurologic examination showed that she had mask face, bradykinesia, bilateral hand tremors of high frequency and low to moderate amplitude, and intensified with postural maintenance. When she rested the hands, tremors were variably and inconsistently present (went away on the left but persisted on the right for a time and then seemed to subside bilaterally) suggesting both rest and action tremors. Her laboratory investigations showed high urea and creatinine, hyperglycemia, hypocalcemia, anemia, and metabolic acidosis [urea: $13 \mathrm{mmol} / \mathrm{l}$; creatinine: $850 \mu \mathrm{mol} / \mathrm{l}$; 
Table 1: Demographic, clinical, laboratory, and imaging characteristics of the studied patients and in relation to the presence or absence of basal ganglia (BG) lesions

\begin{tabular}{|c|c|c|c|c|c|c|}
\hline $\begin{array}{l}\text { Demographic, clinical, laboratory, and neuroimaging } \\
\text { characteristics }\end{array}$ & Total $(n=70)$ & $\begin{array}{c}\text { Patients with } \\
\text { BG lesions } \\
(n=15)\end{array}$ & $\begin{array}{c}\text { Patients without } \\
\text { BG lesions } \\
(n=55) \\
\end{array}$ & $P 1$-value & $P 2$-value & $P 3$-value \\
\hline \multicolumn{7}{|c|}{ Demographic and clinical characteristics } \\
\hline Male & $33(47.1 \%)$ & $4(26.7 \%)$ & $29(52.7 \%)$ & 0.01 & 0.320 & 0.05 \\
\hline Female & $37(52.9 \%)$ & $11(73.3 \%)$ & $26(47.3 \%)$ & 0.01 & 0.335 & 0.01 \\
\hline \multicolumn{7}{|c|}{ Age } \\
\hline Range (years) & $18-64$ & $32-64$ & $18-62$ & & & \\
\hline Mean \pm SD (years) & $48.9 \pm 3.4$ & $55.7 \pm 2.5$ & $46.6 \pm 2.2$ & 0.204 & 0.350 & 0.236 \\
\hline \multicolumn{7}{|c|}{ Duration of uremia } \\
\hline Range (years) & $1-13$ & $1-13$ & $1-11$ & & & \\
\hline Mean \pm SD (years) & $5.5 \pm 1.5$ & $6.3 \pm 1.6$ & $5.3 \pm 1.2$ & 0.456 & 0.875 & 0.325 \\
\hline Not on dialysis & $25(35.7 \%)$ & $7(46.7 \%)$ & $18(32.7 \%)$ & 0.306 & 0.675 & 0.320 \\
\hline Patients on dialysis & $45(64.3 \%)$ & $8(53.3 \%)$ & $37(67.3 \%)$ & 0.356 & 0.768 & 0.247 \\
\hline \multicolumn{7}{|c|}{ Cause of uremia } \\
\hline Glomerulonephritis & $45(64.3 \%)$ & $5(33.3 \%)$ & $40(72.2 \%)$ & 0.001 & 0.220 & 0.0001 \\
\hline Diabetes mellitus & $17(24.3 \%)$ & $9(60 \%)$ & $8(14.5 \%)$ & 0.001 & 0.345 & 0.0001 \\
\hline Congenital kidney agenesis & $4(5.7 \%)$ & $1(6.7 \%)$ & $3(5.5 \%)$ & 0.625 & 0.870 & 0.547 \\
\hline Systemic lupus erythematous (SLE) & $4(5.7 \%)$ & 0 & $4(7.3 \%)$ & - & 0.652 & - \\
\hline \multicolumn{7}{|c|}{ Comorbid medical conditions } \\
\hline Hypertension and ischemic heart disease & $49(70 \%)$ & $6(40 \%)$ & $43(78 \%)$ & 0.001 & 0.763 & 0.001 \\
\hline Diabetes mellitus & $17(24.3 \%)$ & $9(60 \%)$ & $8(14.5 \%)$ & 0.0001 & 0.128 & 0.0001 \\
\hline Hypothyroidism & $2(2.9 \%)$ & 0 & $2(3.6 \%)$ & - & 0.750 & - \\
\hline \multicolumn{7}{|c|}{ Neurologic manifestations } \\
\hline Epilepsy & $11(15.7 \%)$ & & & & & \\
\hline Focal epilepsy to bilateral tonic-clonic seizures & $7(10 \%)$ & $2(13.3 \%)$ & $5(9.1 \%)$ & 0.547 & 0.880 & 0.322 \\
\hline Generalized tonic-clonic epilepsy & $4(5.7 \%)$ & $4(26.7 \%)$ & 0 & 0.01 & - & - \\
\hline Myoclonic brain activity & $33(47 \%)$ & $7(46.7 \%)$ & $26(37.3 \%)$ & 0.657 & 0.468 & 0.001 \\
\hline Epileptic EEG activity in absence of clinical epilepsy & $17(24.3 \%)$ & & $17(30.9 \%)$ & - & 0.352 & - \\
\hline Uremic encephalopathy & $32(45.7 \%)$ & $10(66.7 \%)$ & $22(40 \%)$ & 0.01 & 0.346 & 0.01 \\
\hline Peripheral neuropathy & $47(67.1 \%)$ & $9(60 \%)$ & $38(69.1 \%)$ & 0.342 & 0.532 & 0.322 \\
\hline \multicolumn{7}{|c|}{ Motor manifestations } \\
\hline Asterixis & $42(60 \%)$ & $9(60 \%)$ & $33(60 \%)$ & 1.0 & 1.0 & 1.00 \\
\hline Myoclonus & $48(68.6 \%)$ & $10(66.7 \%)$ & $38(69.1 \%)$ & 0.457 & 0.785 & 0.435 \\
\hline Restless leg syndrome (RLS) & $38(53.3 \%)$ & $12(80 \%)$ & $26(47.3 \%)$ & 0.01 & 0.342 & 0.001 \\
\hline Action tremors & $1(1.4 \%)$ & $1(6.7 \%)$ & 0 & $\mathbf{0 . 0 3}$ & - & - \\
\hline Extrapyramidal movement disorder & $6(8.6 \%)$ & $6(40 \%)$ & 0 & 0.001 & - & - \\
\hline Parkinsonism & $4(5.7 \%)$ & $4(26.7 \%)$ & 0 & 0.001 & - & - \\
\hline Choreo-dystonia & $1(1.4 \%)$ & $1(6.7 \%)$ & 0 & 0.03 & - & - \\
\hline Dystonia & $1(1.4 \%)$ & $1(6.7 \%)$ & 0 & $\mathbf{0 . 0 3}$ & - & - \\
\hline Muscle cramps & $29(41.4 \%)$ & $7(46.7 \%)$ & $22(40 \%)$ & 0.358 & 0.658 & 0.232 \\
\hline Tetany & $6(8.6 \%)$ & $4(26.7 \%)$ & $2(3.6 \%)$ & 0.01 & 0.258 & 0.001 \\
\hline \multicolumn{7}{|c|}{ Laboratory characteristics } \\
\hline$*$ Urea (range) $(\mathrm{mmol} / \mathrm{l})$ & $6.0-35.0$ & $6.0-35.0$ & $7.0-13.0$ & & & \\
\hline *Urea $($ mean+/-SD) $(\mathrm{mmol} / \mathrm{l})$ & $11.0 \pm 3.7$ & $11.4 \pm 2.1$ & $10.0 \pm 1.8$ & 1.0 & 0.876 & 0.563 \\
\hline *Creatinine (range) (umol/l) & $180.0-1472.0$ & $180.0-1472.0$ & $180.0-698.0$ & & & \\
\hline
\end{tabular}


Table 1: (Continued)

\begin{tabular}{|c|c|c|c|c|c|c|}
\hline $\begin{array}{l}\text { Demographic, clinical, laboratory, and neuroimaging } \\
\text { characteristics }\end{array}$ & Total $(n=70)$ & $\begin{array}{c}\text { Patients with } \\
\text { BG lesions } \\
(n=15)\end{array}$ & $\begin{array}{c}\text { Patients without } \\
\text { BG lesions } \\
(n=55)\end{array}$ & $P 1-v a l u e$ & $P 2$-value & P3-value \\
\hline *Creatinine (mean+/-SD) (umol/l) & $309.9 \pm 98.2$ & $520.3 \pm 86.7$ & $253.2 \pm 571$ & 0.01 & 0.230 & 0.001 \\
\hline Hyponatremia & $9(12.86 \%)$ & $5(33.3 \%)$ & $4(7.3 \%)$ & 0.01 & 0.340 & 0.001 \\
\hline Hyperkalemia & $13(18.57 \%)$ & $2(13.3 \%)$ & $11(20 \%)$ & 0.322 & 0.278 & 0.233 \\
\hline Hypomagnesaemia & $8(11.43 \%$ & $1(6.7 \%)$ & $7(12.7 \%)$ & 0.350 & 0.758 & 0.125 \\
\hline Hypocalcemia & $49(70 \%)$ & $15(100 \%)$ & $34(61.8 \%)$ & 0.01 & 0.468 & 0.001 \\
\hline Hyperphosphatemia & $21(30 \%)$ & $7(46.7 \%)$ & $14(25.5 \%)$ & $\mathbf{0 . 0 3}$ & 0.350 & 0.01 \\
\hline Hypoalbuminemia & $29(37.1 \%)$ & $7(46.7 \%)$ & $22(40 \%)$ & 0.242 & 0.478 & 0.458 \\
\hline Low red blood cells (RBCs) count and anemia & $46(68.57 \%)$ & $14(93.3 \%)$ & $32(58.2 \%)$ & 0.001 & 0.126 & 0.001 \\
\hline Hyperglycemia & $17(24.3 \%)$ & $5(33.3 \%)$ & $12(21.8 \%)$ & 0.346 & 0.337 & 0.334 \\
\hline Metabolic acidosis & $24(34.3 \%)$ & $4(26.7 \%)$ & $20(36.4 \%)$ & 0.240 & 0.658 & 0.247 \\
\hline Low thiamine concentrations & $48(68.6 \%)$ & $11(73.3 \%)$ & $37(67.3 \%)$ & 0.320 & 0.673 & 0.352 \\
\hline \multicolumn{7}{|c|}{ Neuroimaging characteristics (CT and/or MRI-brain) } \\
\hline \multicolumn{7}{|c|}{ MRI lesions } \\
\hline White matter hyperintensities (WMHs) & $34(48.6 \%)$ & $9(60 \%)$ & $25(45.5 \%)$ & $\mathbf{0 . 0 3}$ & 0.538 & $\mathbf{0 . 0 2}$ \\
\hline Focal encephalomalacia & $13(18.6 \%)$ & 0 & $13(23.6 \%)$ & - & 0.457 & - \\
\hline Cerebral (lobar) & $12(17.1 \%)$ & 0 & $12(21.8 \%)$ & - & 0.523 & - \\
\hline Thalamic & $1(1.4 \%)$ & 0 & $1(1.8 \%)$ & - & 1.0 & - \\
\hline Basal ganglia lesions & $15(21.4 \%)$ & & & & & \\
\hline Reversible & $3(4.3 \%)$ & $3(20 \%)$ & - & - & - & - \\
\hline Bilateral putamen and globus pallidus lesions & $1(1.4 \%)$ & $1(6.7 \%)$ & - & - & - & - \\
\hline Bilateral putamen and globus pallidus externa lesions & $1(1.4 \%)$ & $1(6.7 \%)$ & - & - & - & - \\
\hline Bilateral lentiform nuclei and thalami lesions & $1(1.4 \%)$ & $1(6.7 \%)$ & - & - & - & - \\
\hline Permanent/residual & $12(17 \%)$ & $12(80 \%)$ & - & - & - & - \\
\hline Right putamen lesions & $3(4.3 \%)$ & $3(20 \%)$ & - & - & - & - \\
\hline Right lentiform lesion & $1(1.4 \%)$ & $1(6.7 \%)$ & - & - & - & - \\
\hline Left caudate lesion & $1(1.4 \%)$ & $1(6.7 \%)$ & - & - & - & - \\
\hline Bilateral lentiform nuclei calcification & $7(10 \%)$ & $7(46.7 \%)$ & & - & - & - \\
\hline
\end{tabular}

Data were expressed as number $(\%)$, range, and mean \pm SD.

Percentage was calculated in relation to the number of patients within the column.

*Urea and creatinine levels for 6 months (median).

$P 1=$ Significance between total studied population and patients with BG lesions; $P 2=$ Significance between total studied population and patients without BG lesions; $P 3=$ Significance between patients with and without BG lesions.

Bold values indicate significance equal to or less than 0.05 .

RBS: $10.5 \mathrm{mmol} / \mathrm{l}$; albumin: $3.4 \mathrm{~g} / \mathrm{l}$; calcium: $1.55 \mathrm{mmol} / \mathrm{l}$ (6.2 $\mathrm{mg} / \mathrm{dl})$; sodium: $136 \mathrm{mmol} / \mathrm{l}$; potassium: $3.6 \mathrm{mmol} / \mathrm{l}$; phosphorus: $4.2 \mathrm{mg} / \mathrm{dl}$; magnesium: $2.1 \mathrm{mg} / \mathrm{dl}$; HGB: $6 \mathrm{mg} / \mathrm{dl}$; RBCs count: 2.5 million/mcl; PH: 7.30; $\mathrm{PO} 2: 95 \mathrm{mmHg}$; $\mathrm{PCO} 2$ : $40 \mathrm{mmHg}$; HCO3: $16 \mathrm{MEq} / \mathrm{l}$; thiamine: $85 \mathrm{nmol} / \mathrm{l}$; venous lactate: $0.88 \mathrm{mmol} / \mathrm{l}]$. Her repeated MRI-brain at the age of 58 was unremarkable (i.e. reversible lesions) (Figure $1(\mathrm{E}-\mathrm{H})$ ).

\section{Case 2}

A 62-year-old man had a 5-year history of T2D causing diabetic nephropathy and uremia at the age of 58 (stage 3; GFR: $55 \mathrm{ml} / \mathrm{min}$ ). He had been on regular treatment with gliclazide
$(60 \mathrm{mg} / \mathrm{d})$ and vitamin B12 (1000 $\mu \mathrm{g} / 5 \mathrm{~d})$. Four months after the onset of uremia, he developed subacute onset of abnormal involuntary movements involving the four limbs (to the same extent) and trunk and sometimes interfered with patient's standing and walking. Neurological consultation confirmed the diagnosis of chorea. There was no history of encephalopathy. His MRI-brain (done within few days after the onset of chorea) showed bilateral symmetrical lesions involved the putamen and the globus pallidus externa which were hypointense in T1WI and hyperintense in T2WI, FLAIR and DWI (restricted motion of water). The ventricles appeared normal and there was no evidence of mass effect (Figure 2 (A-F)). His laboratory 
Table 2: Case reports of basal ganglia (BG) related movement disorders in patients with uremia due to chronic kidney disease

\begin{tabular}{|c|c|c|c|c|c|c|c|}
\hline \multirow{3}{*}{$\begin{array}{l}\text { Patients' } \\
\text { number }\end{array}$} & \multirow{3}{*}{$\begin{array}{l}\text { Age and } \\
\text { sex }\end{array}$} & \multirow{3}{*}{$\begin{array}{l}\text { Comorbid } \\
\text { medical illness }\end{array}$} & \multirow{3}{*}{$\begin{array}{l}\text { Duration of } \\
\text { uremia }\end{array}$} & \multirow{3}{*}{$\begin{array}{l}\text { Duration of } \\
\text { dialysis }\end{array}$} & \multirow{3}{*}{$\begin{array}{l}\text { Duration and } \\
\text { age at onset of } \\
\text { MD }\end{array}$} & $\begin{array}{l}\text { Movement disorder and other } \\
\text { neurologic abnormalities at onset }\end{array}$ & \multirow{3}{*}{ Clinical outcome } \\
\hline & & & & & & $*_{\text {Lab results at onset }}$ & \\
\hline & & & & & & *Neuroimaging at onset & \\
\hline \multirow[t]{15}{*}{1} & \multirow[t]{15}{*}{$58 \mathrm{~F}$} & \multirow{15}{*}{$\begin{array}{l}\text { T2D for } 10 \text { years } \\
\text { (age: } 48 \text { years) }\end{array}$} & \multirow{15}{*}{$\begin{array}{l}3 \text { years (age: } \\
55 \text { years) }\end{array}$} & \multirow{15}{*}{$\begin{array}{l}1 \text { year (age: } \\
56 \text { years) }\end{array}$} & \multirow{15}{*}{$\begin{array}{l}1 \text { year (age: } \\
56 \text { years) }\end{array}$} & At onset: & \multirow{15}{*}{$\begin{array}{l}\text { Progression of Parkinsonism features } \\
\text { (mask face, bilateral rest tremors, and } \\
\text { bradykinesia) and action tremors despite } \\
\text { normalization of MRI-brain }\end{array}$} \\
\hline & & & & & & Rest tremors (Parkinsonian) & \\
\hline & & & & & & Urea $(15 \mathrm{mmol} / \mathrm{l})$ & \\
\hline & & & & & & Creatinine $(1100 \mu \mathrm{mol} / \mathrm{l})$ & \\
\hline & & & & & & Hyperglycemia (15 mmol/l) & \\
\hline & & & & & & Metabolic acidosis (11 MEq/l) & \\
\hline & & & & & & $\begin{array}{l}\text { MRI-brain (within the first week after the } \\
\text { onset): showed lesions in the right } \\
\text { lentiform nucleus, left putamen, and } \\
\text { right occipital lobe which were } \\
\text { hypointense in T1WI, hyperintense in } \\
\text { T2WI, FLAIR, and DWI }\end{array}$ & \\
\hline & & & & & & At period of the study ( 2 years after onset): & \\
\hline & & & & & & $\begin{array}{l}\text { Parkinsonism (mask face, rest tremors, } \\
\text { and bradykinesia) }\end{array}$ & \\
\hline & & & & & & Action tremors (hands) & \\
\hline & & & & & & Urea (13 mmol/l) & \\
\hline & & & & & & Creatinine $(850 \mu \mathrm{mol} / \mathrm{l})$ & \\
\hline & & & & & & Hyperglycemia (10.5 mmol/l) & \\
\hline & & & & & & Metabolic acidosis $(16 \mathrm{MEq} / \mathrm{l})$ & \\
\hline & & & & & & MRI: unremarkable & \\
\hline \multirow[t]{13}{*}{2} & \multirow[t]{13}{*}{$62 \mathrm{M}$} & \multirow{13}{*}{$\begin{array}{l}\text { T2D for } 5 \text { years } \\
\text { (age: } 57 \text { years) }\end{array}$} & \multirow{13}{*}{$\begin{array}{l}4 \text { years (age: } \\
58 \text { years) }\end{array}$} & \multirow[t]{13}{*}{-} & \multirow{13}{*}{$\begin{array}{l}3 \text { years and } \\
8 \text { months (age: } \\
58 \text { years and } 4 \\
\text { months) }\end{array}$} & At onset: & \multirow{13}{*}{$\begin{array}{l}\text { Persistence of choreo-dystonia despite } \\
\text { normalization of MRI-brain } \\
\text { Poor response to treatment with } \\
\text { haloperidol }\end{array}$} \\
\hline & & & & & & Chorea & \\
\hline & & & & & & Urea $(11 \mathrm{mmol} / \mathrm{l})$ & \\
\hline & & & & & & Creatinine $(320 \mu \mathrm{mol} / \mathrm{l})$ & \\
\hline & & & & & & Albumin $(2.6 \mathrm{~g} / \mathrm{l})$ & \\
\hline & & & & & & Hyperglycemia (12 mmol/l) & \\
\hline & & & & & & $\begin{array}{l}\text { MRI-brain (few days after the onset): } \\
\text { showed bilateral symmetrical lesions } \\
\text { involved the putamen and the globus } \\
\text { pallidus externa which were hypointense } \\
\text { in T1WI and hyperintense in T2WI, } \\
\text { FLAIR, and DWI. }\end{array}$ & \\
\hline & & & & & & $\begin{array}{l}\text { MRI-brain (after } 1 \text { month from the onset): } \\
\text { unremarkable }\end{array}$ & \\
\hline & & & & & & $\begin{array}{l}\text { At period of the study ( } 3 \text { years and } \\
8 \text { months after onset): }\end{array}$ & \\
\hline & & & & & & Choreo-dystonia & \\
\hline & & & & & & Urea $(9.3 \mathrm{mmol} / \mathrm{l})$ & \\
\hline & & & & & & Creatinine $(355 \mu \mathrm{mol} / \mathrm{l})$ & \\
\hline & & & & & & MRI: unremarkable & \\
\hline
\end{tabular}


Table 2: (Continued)

\begin{tabular}{|c|c|c|c|c|c|c|c|}
\hline \multirow{3}{*}{$\begin{array}{l}\text { Patients' } \\
\text { number }\end{array}$} & \multirow{3}{*}{$\begin{array}{l}\text { Age and } \\
\text { sex }\end{array}$} & \multirow{3}{*}{$\begin{array}{l}\text { Comorbid } \\
\text { medical illness }\end{array}$} & \multirow{3}{*}{$\begin{array}{l}\text { Duration of } \\
\text { uremia }\end{array}$} & \multirow{3}{*}{$\begin{array}{l}\text { Duration of } \\
\text { dialysis }\end{array}$} & \multirow{3}{*}{$\begin{array}{l}\text { Duration and } \\
\text { age at onset of } \\
\text { MD }\end{array}$} & $\begin{array}{l}\text { Movement disorder and other } \\
\text { neurologic abnormalities at onset }\end{array}$ & \multirow{3}{*}{ Clinical outcome } \\
\hline & & & & & & *Lab results at onset & \\
\hline & & & & & & *Neuroimaging at onset & \\
\hline \multirow[t]{17}{*}{3} & \multirow[t]{17}{*}{$52 \mathrm{~F}$} & \multirow{17}{*}{$\begin{array}{l}\text { HTN for } 20 \text { years } \\
\text { (age: 32years) }\end{array}$} & \multirow{17}{*}{$\begin{array}{l}2 \text { years (age: } \\
50 \text { years) }\end{array}$} & \multirow[t]{17}{*}{-} & \multirow[t]{17}{*}{ De novo } & At onset: & \multirow{17}{*}{$\begin{array}{l}\text { Marked improvement of neurologic } \\
\text { manifestations with hemodialysis, but } \\
\text { persistence of Parkinsonian features and } \\
\text { MRI-brain was unremarkable }\end{array}$} \\
\hline & & & & & & Disorientation & \\
\hline & & & & & & Generalized tonic-clonic & \\
\hline & & & & & & Bradykinesia & \\
\hline & & & & & & Dysarthria & \\
\hline & & & & & & Urea $(10.7 \mathrm{mmol} / \mathrm{l})$ & \\
\hline & & & & & & Creatinine $(550 \mu \mathrm{mol} / \mathrm{l})$ & \\
\hline & & & & & & Sodium (124 mmol/l) & \\
\hline & & & & & & Metabolic acidosis (10 MEq/1) & \\
\hline & & & & & & $\begin{array}{l}\text { MRI-brain ( } 3 \text { days after the onset): } \\
\text { showed bilateral lesions in BG and } \\
\text { thalami which had heterogeneous } \\
\text { intensity in T1WI (hypointense BG and } \\
\text { left thalamic hyperintense lesions) and } \\
\text { hyperintense in T2WI and FLAIR. ADC } \\
\text { map showed that the hyperintense areas } \\
\text { shown in T2WI appeared hyperintense } \\
\text { in their periphery and hypointense in } \\
\text { their centers. The surrounding edema } \\
\text { exerted mass effect on the lateral } \\
\text { ventricles. }\end{array}$ & \\
\hline & & & & & & $\begin{array}{l}\text { At period of the study ( } 1 \text { month after } \\
\text { onset): }\end{array}$ & \\
\hline & & & & & & Parkinsonism & \\
\hline & & & & & & Urea $(9.0 \mathrm{mmol} / \mathrm{l})$ & \\
\hline & & & & & & Creatinine $(320 \mu \mathrm{mol} / \mathrm{l})$ & \\
\hline & & & & & & Sodium $(134 \mathrm{mmol} / \mathrm{l})$ & \\
\hline & & & & & & Metabolic acidosis $(20 \mathrm{MEq} / \mathrm{l})$ & \\
\hline & & & & & & MRI: unremarkable & \\
\hline \multirow[t]{15}{*}{4} & \multirow[t]{15}{*}{$60 \mathrm{~F}$} & \multirow{15}{*}{$\begin{array}{l}\text { T2D for } 8 \text { years } \\
\text { (age: } 52 \text { years) }\end{array}$} & \multirow{15}{*}{$\begin{array}{l}4 \text { years (age: } \\
56 \text { years) }\end{array}$} & \multirow{15}{*}{$\begin{array}{l}2 \text { years (age: } \\
58 \text { years) }\end{array}$} & \multirow{15}{*}{$\begin{array}{l}1 \text { years and } \\
9 \text { months (age: } \\
58 \text { years and } \\
3 \text { months) }\end{array}$} & At onset: & \multirow{15}{*}{$\begin{array}{l}\text { Progression of Parkinsonism } \\
\text { Partial improvement of levodopa/ } \\
\text { carbidopa }\end{array}$} \\
\hline & & & & & & Parkinsonism & \\
\hline & & & & & & Urea $(11 \mathrm{mmol} / \mathrm{l})$ & \\
\hline & & & & & & Creatinine $(360 \mu \mathrm{mol} / \mathrm{l})$ & \\
\hline & & & & & & Hyperglycemia (11 mmol/l) & \\
\hline & & & & & & Sodium (124 mmol/l) & \\
\hline & & & & & & Metabolic acidosis $(10 \mathrm{MEq} / \mathrm{l})$ & \\
\hline & & & & & & $\begin{array}{l}\text { Computed tomography (CT)-brain: } \\
\text { unremarkable }\end{array}$ & \\
\hline & & & & & & $\begin{array}{l}\text { At period of the study ( } 2 \text { years and } \\
3 \text { months after onset): }\end{array}$ & \\
\hline & & & & & & Parkinsonism & \\
\hline & & & & & & Urea $(12 \mathrm{mmol} / \mathrm{l})$ & \\
\hline & & & & & & Creatinine $(526 \mu \mathrm{mol} / \mathrm{l})$ & \\
\hline & & & & & & Hyperglycemia $(11 \mathrm{mmol} / \mathrm{l})$ & \\
\hline & & & & & & Metabolic acidosis (17 MEq/1) & \\
\hline & & & & & & $\begin{array}{l}\text { MRI-brain: a lesion in the right putamen } \\
\text { and globus pallidus externa which was } \\
\text { hyperintense in FLAIR and ADC (focal } \\
\text { encephalomalacia) and WMHs }\end{array}$ & \\
\hline
\end{tabular}


Table 2: (Continued)

\begin{tabular}{|c|c|c|c|c|c|c|c|}
\hline \multirow{3}{*}{$\begin{array}{l}\text { Patients' } \\
\text { number }\end{array}$} & \multirow{3}{*}{$\begin{array}{l}\text { Age and } \\
\text { sex }\end{array}$} & \multirow{3}{*}{$\begin{array}{l}\text { Comorbid } \\
\text { medical illness }\end{array}$} & \multirow{3}{*}{$\begin{array}{l}\text { Duration of } \\
\text { uremia }\end{array}$} & \multirow{3}{*}{$\begin{array}{l}\text { Duration of } \\
\text { dialysis }\end{array}$} & \multirow{3}{*}{$\begin{array}{l}\text { Duration and } \\
\text { age at onset of } \\
\text { MD }\end{array}$} & $\begin{array}{l}\text { Movement disorder and other } \\
\text { neurologic abnormalities at onset }\end{array}$ & \multirow{3}{*}{ Clinical outcome } \\
\hline & & & & & & *Lab results at onset & \\
\hline & & & & & & *Neuroimaging at onset & \\
\hline \multirow[t]{11}{*}{5} & \multirow[t]{11}{*}{$58 \mathrm{~F}$} & \multirow{11}{*}{$\begin{array}{l}\text { T2D for } 10 \text { years } \\
\text { (age: } 48 \text { years) } \\
\text { HTN for } 3 \text { years } \\
\text { (age: } 55 \text { years) }\end{array}$} & \multirow{11}{*}{$\begin{array}{l}3 \text { years (age: } \\
55 \text { years) }\end{array}$} & \multirow[t]{11}{*}{-} & \multirow{11}{*}{$\begin{array}{l}2 \text { years and } \\
2 \text { months (age: } \\
55 \text { years and } \\
10 \text { months) }\end{array}$} & At onset: & \multirow{11}{*}{$\begin{array}{l}\text { Persistence of Parkinsonism } \\
\text { Right lentiform nucleus } \\
\text { encephalomalacia } \\
\text { Poor response to levodopa/carbidopa }\end{array}$} \\
\hline & & & & & & Parkinsonism & \\
\hline & & & & & & Urea $(7.5 \mathrm{mmol} / \mathrm{l})$ & \\
\hline & & & & & & Creatinine $(250 \mu \mathrm{mol} / \mathrm{l})$ & \\
\hline & & & & & & $\begin{array}{l}\text { MRI-brain: a lesion in the right lentiform } \\
\text { nucleus which was hypointense in T1WI } \\
\text { and ADC and hyperintense in T2WI and } \\
\text { FLAIR }\end{array}$ & \\
\hline & & & & & & $\begin{array}{l}\text { At period of the study ( } 2 \text { years and } \\
2 \text { months after onset): }\end{array}$ & \\
\hline & & & & & & Parkinsonism & \\
\hline & & & & & & Urea $(7.5 \mathrm{mmol} / \mathrm{l})$ & \\
\hline & & & & & & Creatinine $(250 \mu \mathrm{mol} / \mathrm{l})$ & \\
\hline & & & & & & Hyperglycemia $(8.5 \mathrm{mmol} / \mathrm{l})$ & \\
\hline & & & & & & $\begin{array}{l}\text { MRI-brain: a small right hyperintense } \\
\text { lesion in FLAIR and hypointense in DWI }\end{array}$ & \\
\hline \multirow[t]{16}{*}{6} & \multirow[t]{16}{*}{$60 \mathrm{~F}$} & \multirow{16}{*}{$\begin{array}{l}\text { HTN for } 8 \text { years } \\
\text { (age: } 52 \text { years) }\end{array}$} & \multirow{16}{*}{$\begin{array}{l}6 \text { years (age: } \\
54 \text { years) }\end{array}$} & \multirow{16}{*}{$\begin{array}{l}3 \text { years (age: } \\
57 \text { years }\end{array}$} & \multirow{16}{*}{$\begin{array}{l}5 \text { years and } \\
2 \text { months (age: } \\
54 \text { years and } \\
10 \text { months) }\end{array}$} & At onset: & \multirow{16}{*}{$\begin{array}{l}\text { Persistence of dystonia } \\
\text { Right putamen encephalomalacia and } \\
\text { WMHs }\end{array}$} \\
\hline & & & & & & Uremic encephalopathy & \\
\hline & & & & & & Myoclonic jerks & \\
\hline & & & & & & Focal jaw dystonia & \\
\hline & & & & & & Urea $(12 \mathrm{mmol} / \mathrm{l})$ & \\
\hline & & & & & & Creatinine $(860 \mu \mathrm{mol} / \mathrm{l})$ & \\
\hline & & & & & & Sodium $(124 \mathrm{mmol} / \mathrm{l})$ & \\
\hline & & & & & & Metabolic acidosis $(10 \mathrm{MEq} / \mathrm{l})$ & \\
\hline & & & & & & $\begin{array}{l}\text { CT-brain: right putamen hypodense } \\
\text { lesion }\end{array}$ & \\
\hline & & & & & & $\begin{array}{l}\text { At period of the study ( } 5 \text { years and } \\
2 \text { months after onset): }\end{array}$ & \\
\hline & & & & & & Focal jaw dystonia & \\
\hline & & & & & & Urea $(11.8 \mathrm{mmol} / \mathrm{l})$ & \\
\hline & & & & & & Creatinine $(780 \mu \mathrm{mol} / \mathrm{l})$ & \\
\hline & & & & & & Sodium $(129 \mathrm{mmol} / \mathrm{l})$ & \\
\hline & & & & & & Metabolic acidosis (17 MEq/l) & \\
\hline & & & & & & $\begin{array}{l}\text { MRI-brain: hyperintense lesion in T2WI } \\
\text { and FLAIR similar to CT findings and } \\
\text { discrete subcortical WMHs in both } \\
\text { hemispheres }\end{array}$ & \\
\hline
\end{tabular}

$\mathrm{ND}=$ not determined $\mathrm{F}=$ female $\mathrm{M}=$ male $\mathrm{T} 2 \mathrm{D}=$ type 2 diabetes $\mathrm{HTN}=$ hypertension; $\mathrm{MD}=$ movement disorder; $\mathrm{BG}=$ basal ganglia; $\mathrm{T} 1 \mathrm{WI}=\mathrm{T} 1$ weighted image; T2WI = T2-weighted; FLAIR = fluid attenuated inversion recovery; DWI = diffusion-weighted image; ADC $=$ apparent diffusion coefficient; WMHs = white matter hyperintensities.

*At onset of neurologic condition.

investigations were significant for high urea and creatinine, hyperglycemia, hypoalbuminemia, hypocalcemia, and anemia [urea: $11 \mathrm{mmol} / \mathrm{l}$; creatinine: $320 \mu \mathrm{mol} / \mathrm{l}$; RBS: $12 \mathrm{mmol} / \mathrm{l}$; albumin: $2.6 \mathrm{~g} / \mathrm{l}$; calcium: $7.2 \mathrm{mg} / \mathrm{dl}$ ( $1.8 \mathrm{mmol} / \mathrm{l})$; sodium: $136 \mathrm{mmol} / \mathrm{l}$; potassium: $3.6 \mathrm{mmol} / \mathrm{l}$; phosphorus: $4.2 \mathrm{mg} / \mathrm{dl}$; magnesium: $2.1 \mathrm{mg} / \mathrm{dl}$; HGB: $8.5 \mathrm{mg} / \mathrm{dl}$; RBCs count: $3.2 \mathrm{million} / \mathrm{mcl}$;
PH: 7.30; PO2: 95 mmHg; PCO2: 40 mmHg; HCO3: $21 \mathrm{MEq} / \mathrm{l}]$. Clinical and neuroimaging follow-ups were done 1 month after the onset and showed persistence of chorea despite complete resolution of MRI findings (i.e. reversible lesions). Afterward, the patient did multiple neurologic consultations and MRI-brains which showed similar findings. Treatment with haloperidol 


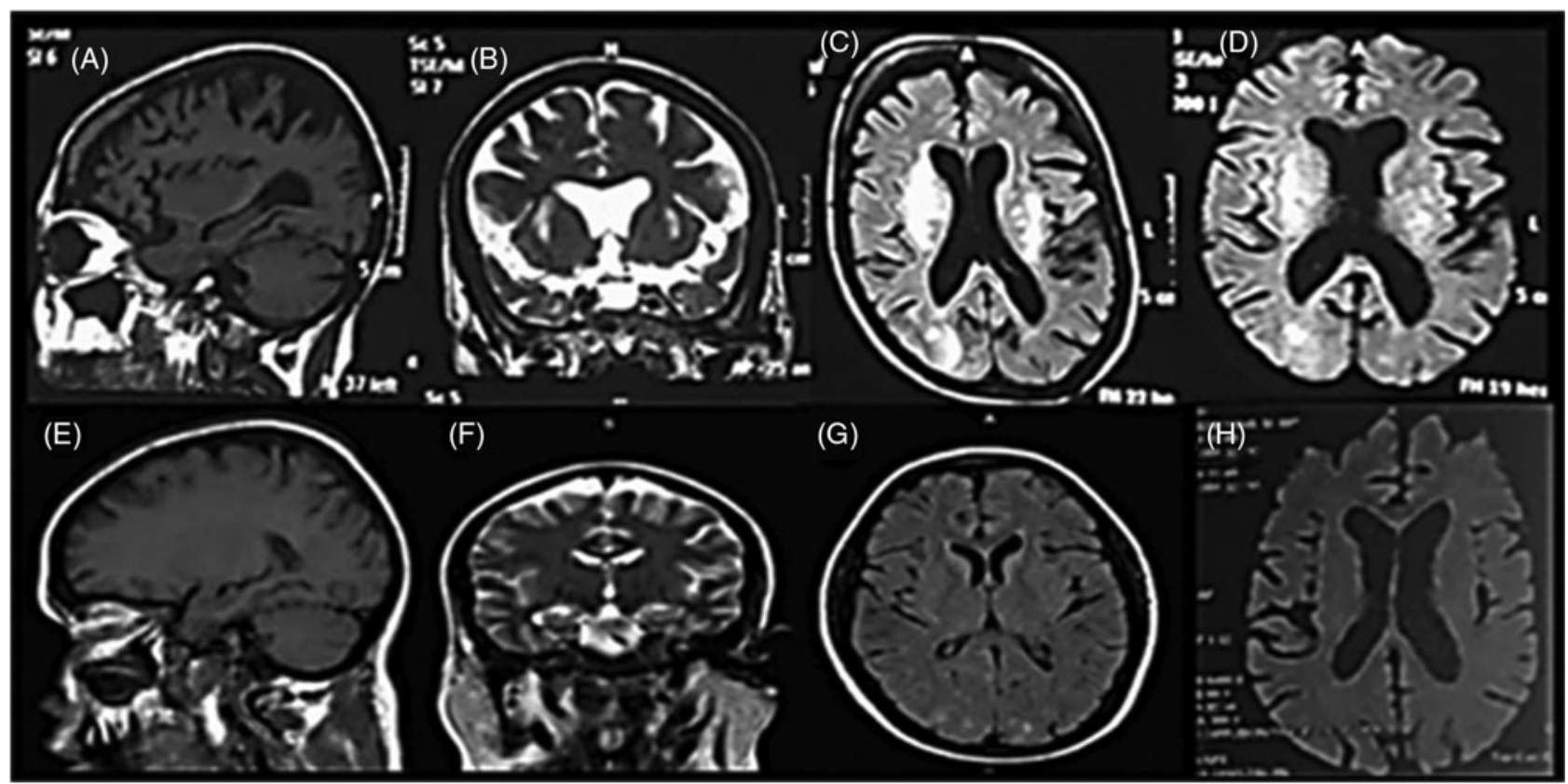

Figure 1: MRI-brain views (sagittal, coronal, and axial) of Case (1) with Parkinsonism. They showed lesions in BG (right lentiform nucleus and left putamen) and right occipital lobe. They appeared hypointense in T1WI (A) and hyperintense in T2WI (B), FLAIR (C), and DWI (D). Repeated MRI-brain views (2 years after onset) were unremarkable (i.e. reversible lesions) $(E-H)$.

provided no benefit and the patient denied deterioration of his condition over years. Evaluation at the age of 62 years (the period of the study) showed an alert and cooperative male. He had no history of specific drug intake other than treatment of diabetes and oral supplements of calcium and vitamin D. He had never been on dialysis. His blood pressure was $140 / 80 \mathrm{mmHg}$, heart rate was 90 beats/min, respiratory rate was 24 breaths/min, and temperature was $37.2^{\circ} \mathrm{C}$. There were irregular, arrhythmic, continuous, and rapid involuntary movements in the distal limbs characteristic of chorea. He also had chorea of the proximal legs, pelvis, and trunk as well as dystonia in the right arm. There were no facial involuntary movements. His laboratory investigations showed high urea and creatinine, hyperglycemia, hypoalbuminemia, hypocalcemia, anemia, and low thiamine concentration [urea: $9.3 \mathrm{mmol} / \mathrm{l}$; creatinine: $355 \mu \mathrm{mol} / \mathrm{l}$; RBS: $7 \mathrm{mmol} / \mathrm{l}$; albumin: $3.0 \mathrm{~g} / \mathrm{l}$; calcium: $1.925 \mathrm{mmol} / \mathrm{l}(7.7 \mathrm{mg} / \mathrm{dl})$; sodium: $134 \mathrm{mmol} / \mathrm{l}$; potassium: $3.6 \mathrm{mmol} / \mathrm{l}$; phosphorus: $3.8 \mathrm{mg} / \mathrm{dl}$; magnesium: $2.0 \mathrm{mg} / \mathrm{dl}$; HGB: $7.2 \mathrm{mg} / \mathrm{dl}$; RBCs count: $3.0 \mathrm{million} / \mathrm{mcl}$; $\mathrm{PH}$ : 7.3; PO2: 90 mmHg; PCO2: 40 mmHg; HCO3: $20 \mathrm{MEq} /$; thiamine: $42 \mathrm{nmol} / \mathrm{l}$; venous lactate: $0.58 \mathrm{mmol} / \mathrm{l}]$. His repeated MRI-brain (at the age of 62) was unremarkable (Figure $2(\mathrm{G}-\mathrm{I})$ ).

\section{Case 3}

A 52-year-old female had a 20-year history of hypertension due to congenital renal agenesis and developed uremia at the age of 50 (stage 3; GFR: $58 \mathrm{ml} / \mathrm{min}$ ). She had been on regular treatment with amlodipine and valsartan for hypertension, dietary control, and supportive treatment. There was no history of encephalopathy. She had never been on dialysis. Neurologic consultation was done to the patient (during the period of our study) because she developed acute onset of recurrent three attacks of generalized tonic-clonic convulsions, disorientation, slowness of movement (bradykinesia), fatigue, and dysarthria. Her consciousness was intact in between the attacks of seizures and had intact movement of the four limbs. Her computed tomography (CT) of the brain was unremarkable (done within the first $24 \mathrm{~h}$ after the insult). Her laboratory investigations showed very higher levels of urea and creatinine compared to her baseline, hyponatremia, and metabolic acidosis [urea: $10.7 \mathrm{mmol} / \mathrm{l}$; creatinine: $550 \mu \mathrm{mol} / \mathrm{l}$; RBS: $5 \mathrm{mmol} / \mathrm{l}$; albumin: $3.4 \mathrm{~g} / \mathrm{l}$; calcium: $2.5 \mathrm{mmol} / \mathrm{l}(10 \mathrm{mg} / \mathrm{dl})$; sodium: $124 \mathrm{mmol} / 1$; potassium: $3.3 \mathrm{mmol} / 1$; phosphorus: $4.5 \mathrm{mg} / \mathrm{dl}$; magnesium: $2.6 \mathrm{mg} / \mathrm{dl}$; HGB: $10.4 \mathrm{mg} / \mathrm{dl}$; RBCs count: 3.6 million/mcl; PH: 7.34; PO2: $70 \mathrm{mmHg}$; PCO2: $46 \mathrm{mmHg}$; HCO3: $10 \mathrm{MEq} / \mathrm{l}$; thiamine: $90 \mathrm{nmol} / \mathrm{l}$; venous lactate: $0.64 \mathrm{mmol} / \mathrm{l}]$. Her MRI-brain (Figure 3) (done 3 days after the insult) showed bilateral lesions in BG and thalami which had heterogeneous intensity in T1WI (hypointense BG and left thalamic hyperintense lesions) (A, B, and C) and hyperintense in T2WI (D) and FLAIR (E). Apparent diffusion coefficient (ADC) map (F) showed that the hyperintense areas shown in T2WI appeared hyperintense in their periphery (suggesting free motion of water of vasogenic edema) and hypointense in their centers (suggesting restricted motion of water or cytotoxic edema) $(\mathrm{G}$ and $\mathrm{H})$. The surrounding edema exerted mass effect on the lateral ventricles but no midline shift. Emergency hemodialysis was done (three times per week) for 3 weeks which resulted in reduction of urea and creatinine levels (urea: $9.0 \mathrm{mmol} / \mathrm{l}$; creatinine: $320 \mu \mathrm{mol} / \mathrm{l}$; sodium: 134 mmol/l; PH: 7.32; PO2: 70 mmHg; PCO2: 44 mmHg; HCO3: $21 \mathrm{MEq} / \mathrm{l})$. The reduction of severe uremia and correction of metabolic abnormalities were associated with marked clinical improvement and resolution of MRI findings within 4 weeks. She did not receive hemodialysis afterward. Follow-up showed persistence of manifestations of Parkinsonism (mask face and bradykinesia). Her repeated MRI-brain (a year later during the study period) was unremarkable (I-L). 


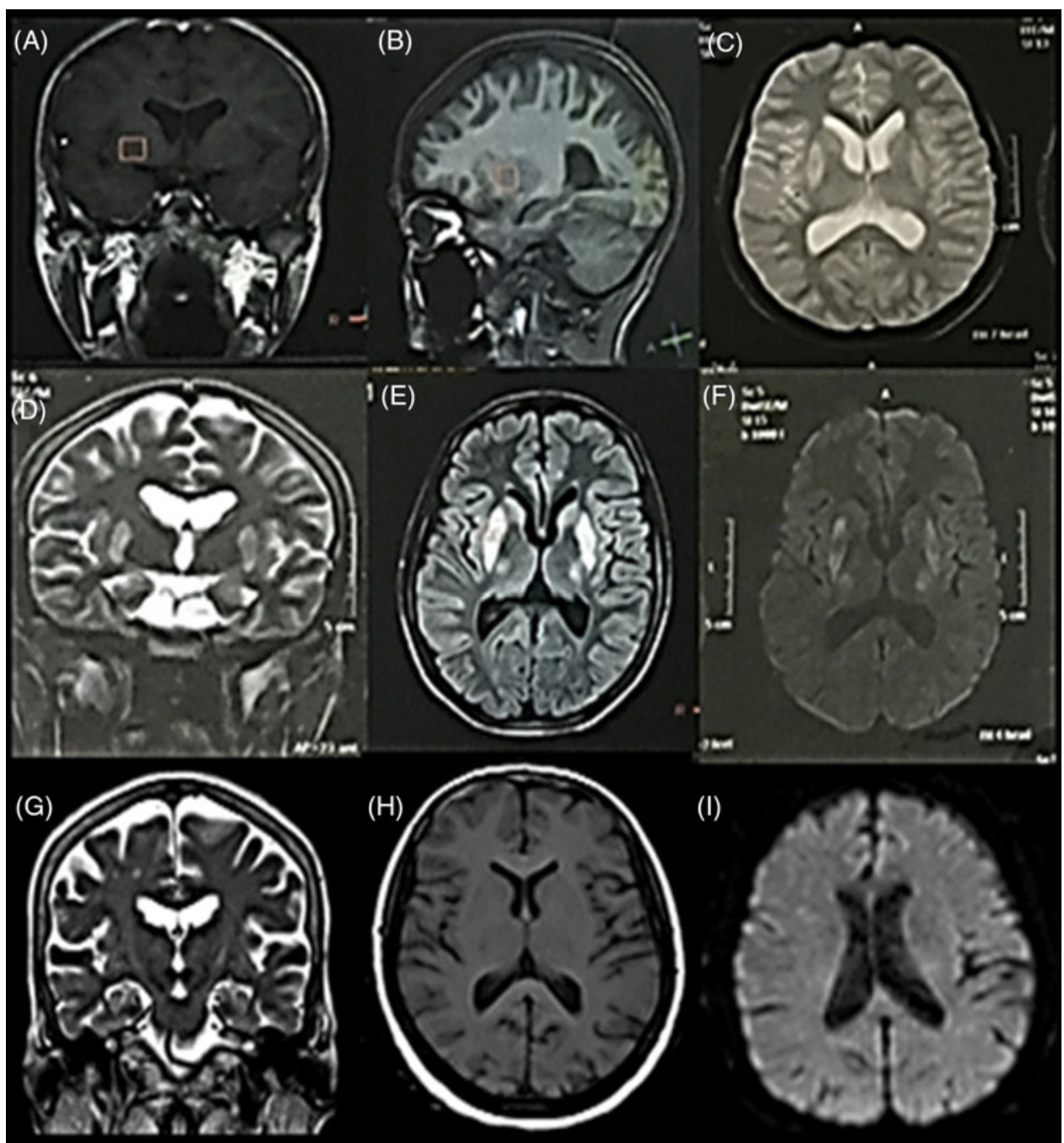

Figure 2: MRI-brain views (coronal, sagittal, and axial) of Case 2 with choreo-dystonia. They showed bilateral BG lesions (putamen and globus pallidus externa) which were hypointense in TIWI (A and B) and hyperintense in T2WI (C and D), FLAIR $(E)$, and DWI $(F)$. Repeated MRI-brain (after 3 years and 8 months of the neurologic condition) $(G-I)$ were unremarkable (i.e. reversible lesions).

\section{Case 4}

A 60-year-old female had an 8-year history of T2D causing diabetic nephropathy and uremia (stage 4; GFR: $29 \mathrm{ml} / \mathrm{min}$ ) at the age of 56 . She had been on regular treatment with gliclazide $(60 \mathrm{mg} / \mathrm{d})$, vitamin B12 $(1000 \mu \mathrm{g} / 3 \mathrm{~d})$, and regular hemodialysis (three times per week with session duration of 2-3 h) for 2 years. Three months after development of uremia, she developed rapid onset (within several weeks) of rest tremors in her left hand. There was no history of uremic encephalopathy. She underwent neurologic consultation and diagnosed as Parkinsonism. Her CT brain showed no abnormalities. Her laboratory investigations were significant for high urea and creatinine, hyperglycemia, hypoalbuminemia hypocalcemia, hyponatremia, anemia, and metabolic acidosis [urea: $11 \mathrm{mmol} / \mathrm{l}$; creatinine: $360 \mu \mathrm{mol} / \mathrm{l}$; RBS: $11 \mathrm{mmol} / \mathrm{l}$; albumin: $2.8 \mathrm{~g} / \mathrm{l}$; calcium: $1.8 \mathrm{mmol} / 1$ (7.2 mg/dl); sodium: $124 \mathrm{mmol} / \mathrm{l}$; potassium: $3.3 \mathrm{mmol} / \mathrm{l}$; phosphorus: $4.5 \mathrm{mg} / \mathrm{dl}$; magnesium: $2.6 \mathrm{mg} / \mathrm{dl}$; HGB: $8.0 \mathrm{mg} / \mathrm{dl}$; RBCs count: $3.5 \mathrm{million} /$ mcl; PH: 7.3; PO2: 77 mmHg; PCO2: 40 mmHg; HCO3: $10 \mathrm{MEq} / \mathrm{l}]$. Over the next one and half year, she noticed progression of her condition as tremors became worse and involved her both hands (left $>$ right). She also developed bradykinesia and shortstepped gait. She showed partial improvement with levodopa/ carbidopa. Neurological evaluation (age: 60 years old, during the period of the study) showed an alert, cooperative female. Her blood pressure was $130 / 80 \mathrm{mmHg}$, heart rate was 98 beats/min, respiratory rate was 28 breaths $/ \mathrm{min}$, and temperature was $37.0^{\circ} \mathrm{C}$. She had mask face, rest tremors in both hands (left $>$ right), and high frequency low amplitude side to side rest tremors of head, low monotonous speech, bradykinesia, limb rigidity, and peripheral neuropathy (motor and sensory). Her laboratory investigations showed high urea and creatinine, hyperglycemia, hypoalbuminemia, hypocalcemia; anemia, metabolic acidosis, and low thiamine concentration [urea: $12 \mathrm{mmol} / \mathrm{l}$; creatinine: $526 \mu \mathrm{mol} / \mathrm{l}$; RBS: $11 \mathrm{mmol} / \mathrm{l}$; albumin: $2.7 \mathrm{~g} / \mathrm{l}$; calcium: $7 \mathrm{mg} / \mathrm{dl}$ ( $1.75 \mathrm{mmol} / \mathrm{l})$; sodium: $132 \mathrm{mmol} / \mathrm{l}$; potassium: $4.2 \mathrm{mmol} / \mathrm{l}$; phosphorus: $3.6 \mathrm{mg} / \mathrm{dl}$; magnesium: $1.7 \mathrm{mg} / \mathrm{dl}$; HGB: $8.3 \mathrm{mg} / \mathrm{dl}$; RBCs count: 3.8 million/mcl; PH: 7.32; PO2: $88 \mathrm{mmHg}$; PCO2: $36 \mathrm{mmHg}$; HCO3: $17 \mathrm{MEq} / \mathrm{l}$; thiamine: $44 \mathrm{nmol} / \mathrm{l}$; venous lactate: $0.67 \mathrm{mmol} / \mathrm{l}]$. Her MRI-brain (age: 60 years) (Figure 4) showed a lesion in the right putamen and globus pallidus externa 


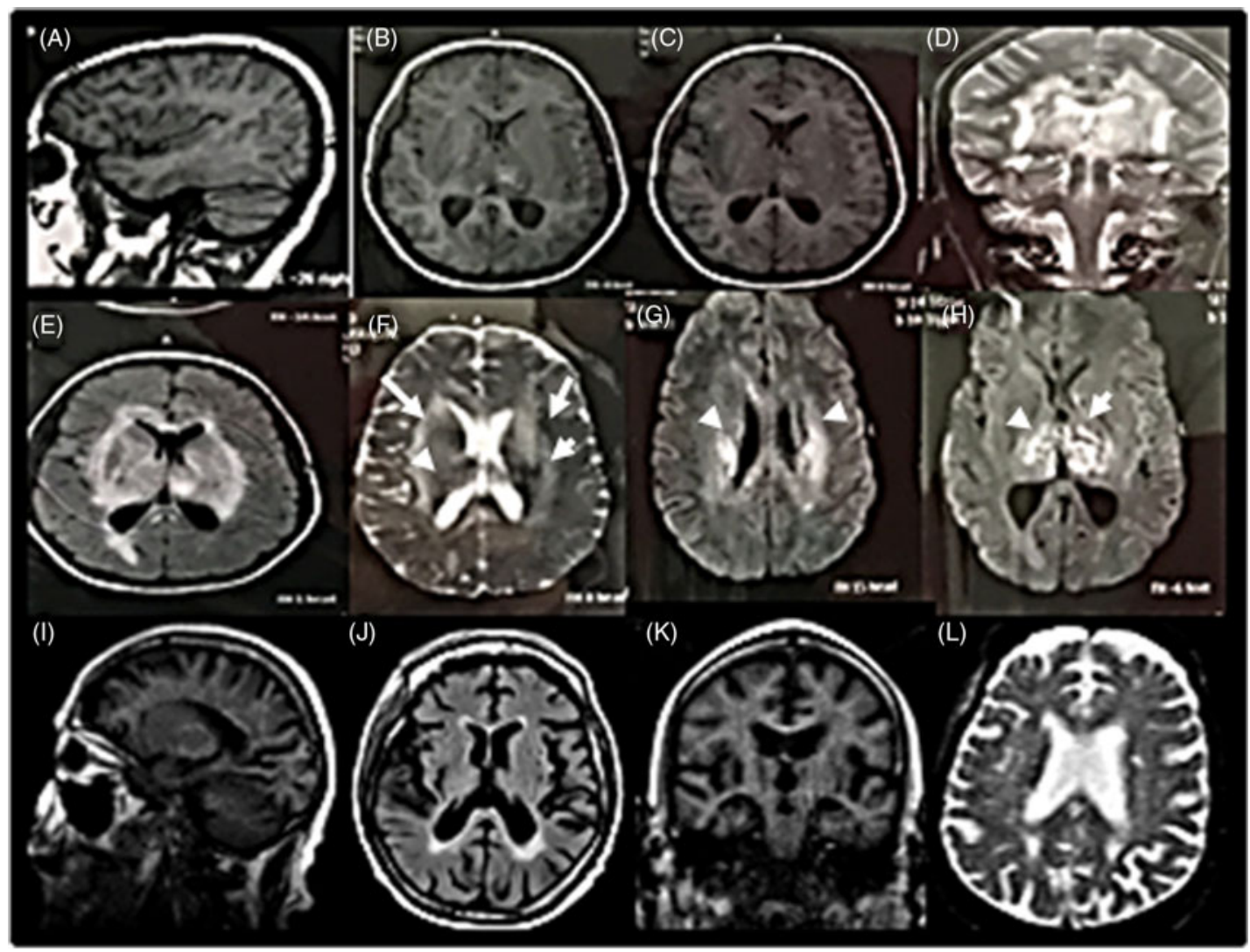

Figure 3: MRI-brain views (sagittal, axial, and coronal) of Case 3 with Parkinsonism. They showed bilateral BG (lentiform nuclei) lesions which have heterogeneous intensity in TIWI (hypointense BG and left hyperintense thalamic lesions) $(A-C)$ and marked hyperintense in T2WI $(D)$ and FLAIR $(E)$. ADC map $(F)$ showed that the hyperintense areas shown in T2WI had peripheral hyperintensities (suggesting free motion of water of vasogenic edema) (arrows) and more central hypointense areas. The latter appeared as two hyperintense areas (suggesting restricted motion of water or cytotoxic edema) (arrowheads) ( $G$ and $H$ ). MRI-brain views after 4 weeks ( $I-L)$ were unremarkable (i.e. reversible lesions).

which was hyperintense in FLAIR (A) and ADC (B) or CSF like and dilated adjacent lateral ventricle (suggesting focal encephalomalacia) and bilateral periventricular small white matter lesions which were hypointense in T1WI (C) and hyperintense in FLAIR (D) and T2WI (WMHs) (E and F).

\section{Case 5}

A 58-year-old female had a 10-year history of T2D causing diabetic nephropathy and uremia (stage 3; GFR; $45 \mathrm{ml} / \mathrm{min}$ ) at the age of 55. She had comorbid hypertension for 3 years. She had been on treatment with gliclazide $(60 \mathrm{mg} / \mathrm{d})$, lisinopril, and vitamin B12 $(1000 \mu \mathrm{g} / 5 \mathrm{~d})$. Ten months after the onset of uremia, she developed acute manifestations of bradykinesia, left limb rigidity, and rest tremors. Neurological consultation confirmed the diagnosis of Parkinsonism. Her lab investigations were only significant for high urea and creatinine [urea: $7.8 \mathrm{mmol} / \mathrm{l}$; creatinine: $250 \mu \mathrm{mol} / \mathrm{l}$; RBS: $7.0 \mathrm{mmol} / \mathrm{l}$; albumin: $3.3 \mathrm{~g} / \mathrm{l}$; calcium: $10.0 \mathrm{mg} / \mathrm{dl}$ (2.5 mmol/l); sodium: $132 \mathrm{mmol} / \mathrm{l}$; potassium: $4.0 \mathrm{mmol} / \mathrm{l}$; phosphorus: $4.2 \mathrm{mg} / \mathrm{dl}$; magnesium: $1.6 \mathrm{mg} / \mathrm{dl}$; HGB: $8.5 \mathrm{mg} / \mathrm{dl}$; RBCs count: $3.0 \mathrm{million} / \mathrm{mcl}$; $\mathrm{PH}$ : 7.30; PO2: $88 \mathrm{mmHg}$; PCO2: $40 \mathrm{mmHg}$; and HCO3: $22 \mathrm{MEq} / \mathrm{l}]$. Her MRI-brain (Figure 5) (done within the first week after the onset) showed a lesion in the right lentiform nucleus which was hypointense in ADC (A) and hyperintense in T2WI (B) and
FLAIR (C). She had poor response to levodopa/carbidopa. Neurological evaluation (at the age of 58, during the study period) showed an alert and cooperative female. She had history of RLS. She denied deterioration of her neurologic condition. Her blood pressure was $130 / 85 \mathrm{mmHg}$, heart rate was 90 beats/min, respiratory rate was 24 breaths/min, and temperature was $36.6^{\circ} \mathrm{C}$. She had mask face, bradykinesia, limb rigidity, and rest tremors (left $>$ right). She had sensory neuropathy in her lower limbs. Her laboratory investigations showed high urea and creatinine, hyperglycemia, anemia, and low thiamine level [urea: $7.6 \mathrm{mmol} / \mathrm{l}$; creatinine: $250 \mu \mathrm{mol} / \mathrm{l}$; RBS: $8.5 \mathrm{mmol} / \mathrm{l}$; albumin: $3.4 \mathrm{~g} / \mathrm{l}$; calcium: $2.5 \mathrm{mmol} / \mathrm{l}$ (9.0 mg/dl); sodium: $134 \mathrm{mmol} / \mathrm{l}$; potassium: $4.2 \mathrm{mmol} / \mathrm{l}$; phosphorus: $3.8 \mathrm{mg} / \mathrm{dl}$; magnesium: $1.8 \mathrm{mg} / \mathrm{dl}$; HGB: $8.5 \mathrm{mg} / \mathrm{dl}$; RBCs count: 3.0 million/mcl; PH: 7.30; PO2: $80 \mathrm{mmHg}$; PCO2: $44 \mathrm{mmHg}$; HCO3: $21 \mathrm{MEq} / \mathrm{l}$; thiamine: $50 \mathrm{nmol} / \mathrm{l}$; venous lactate: $0.58 \mathrm{mmol} / \mathrm{l}]$. MRI-brain (done 2 years and 2 months after the onset) showed marked reduction of the lesion size to a small right hyperintense lesion in FLAIR (D) and hypointense in DWI (E) (Figure 5).

\section{Case 6}

A 60-year-old female had an 8-year history of hypertension and uremia due to chronic nephritis (stage 5; GFR: $12 \mathrm{ml} / \mathrm{min}$ ) at 


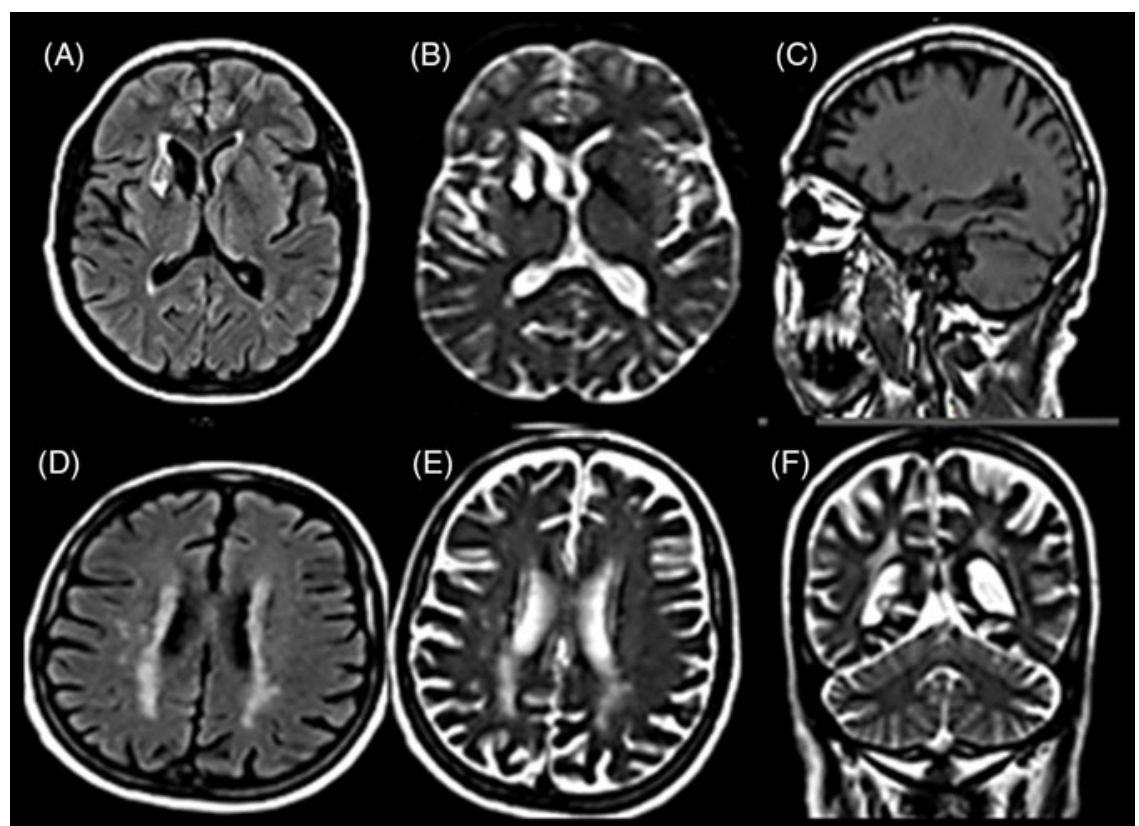

Figure 4: MRI-brain views (axial and coronal) of Case 4 with Parkinsonism. There was hyperintense lesion in the right putamen and globus pallidus externa in FLAIR $(A)$ and $A D C(C)$ (focal encephalomalacia). There were also bilateral subcortical small white matter lesions particularly periventricular which were hypointense in $T 1 W I(C)$ and hyperintense in FLAIR $(D)$ and T2WI (WMHs) (E and F).

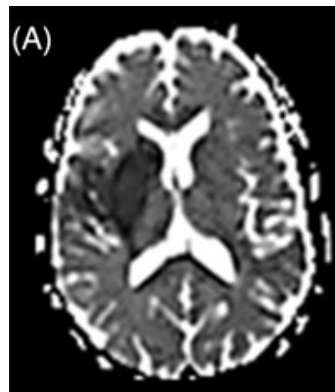

(B)

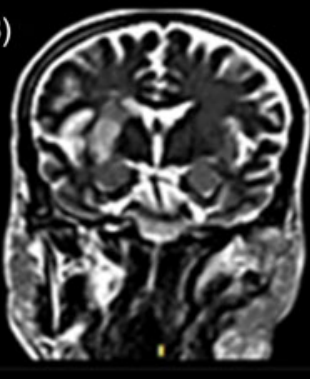

(C)
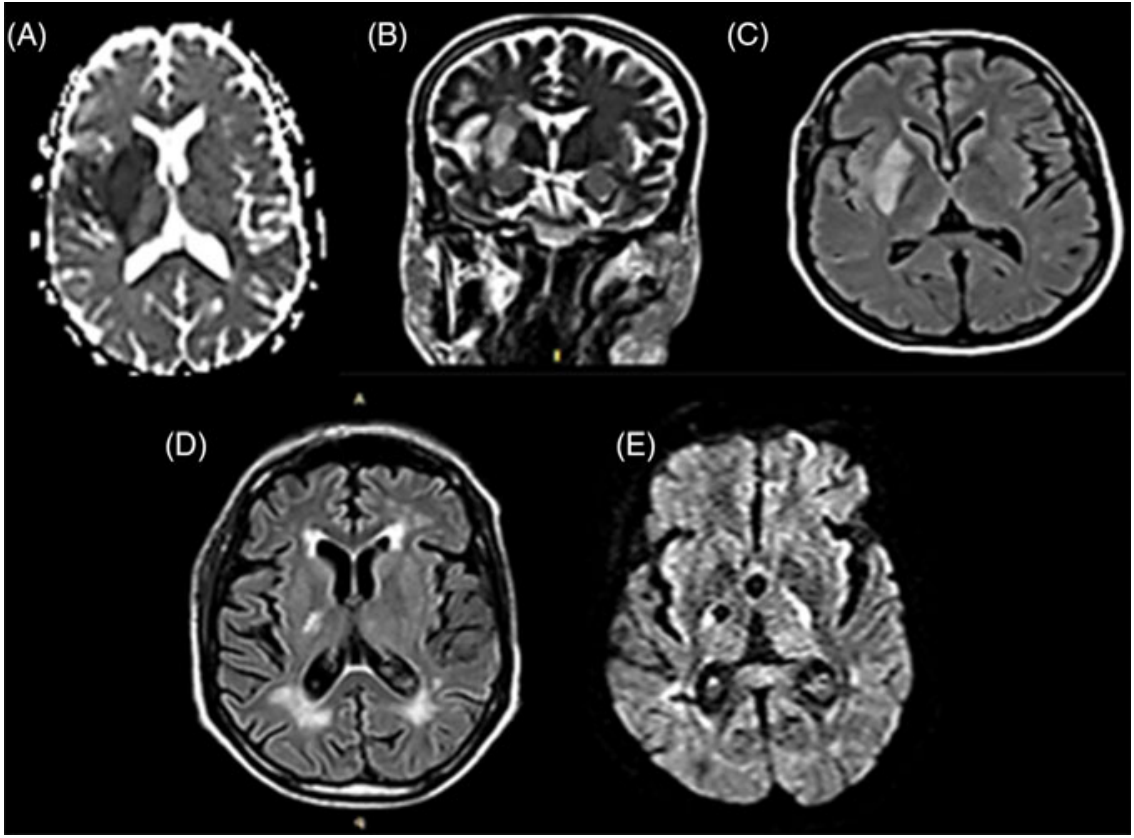

Figure 5: MRI-brain views (coronal and axial) of Case 5 with Parkinsonism. They showed a lesion in the right lentiform nucleus which was hypointense in $A D C(A)$ and hyperintense in T2WI $(B)$ and FLAIR $(C)$. Repeated MRI-brain (2 years and 2 months after the onset) showed marked reduction of the lesion size to a small right hyperintense lesion shown in FLAIR (D) and hypointense in $D W I(E)$.

the age of 54. She had been on regular treatment with Co-Diovan $160 / 25 \mathrm{mg}$ (160 $\mathrm{mg}$ valsartan and $25 \mathrm{mg}$ hydrochlorothiazide). She had been on hemodialysis for 3 years (1-3 times per week with session duration of $1.5-2.5 \mathrm{~h}$ ). Approximately 10 months after the onset of uremia, she developed attacks of uremic encephalopathy and episodes of abnormal involuntary movements which were marked in the face. CT-brain showed hypodense lesion in the right putamen (Figure 6A). Her laboratory investigations were significant for high urea and creatinine, hyponatremia, metabolic acidosis, hypocalcemia, and anemia 


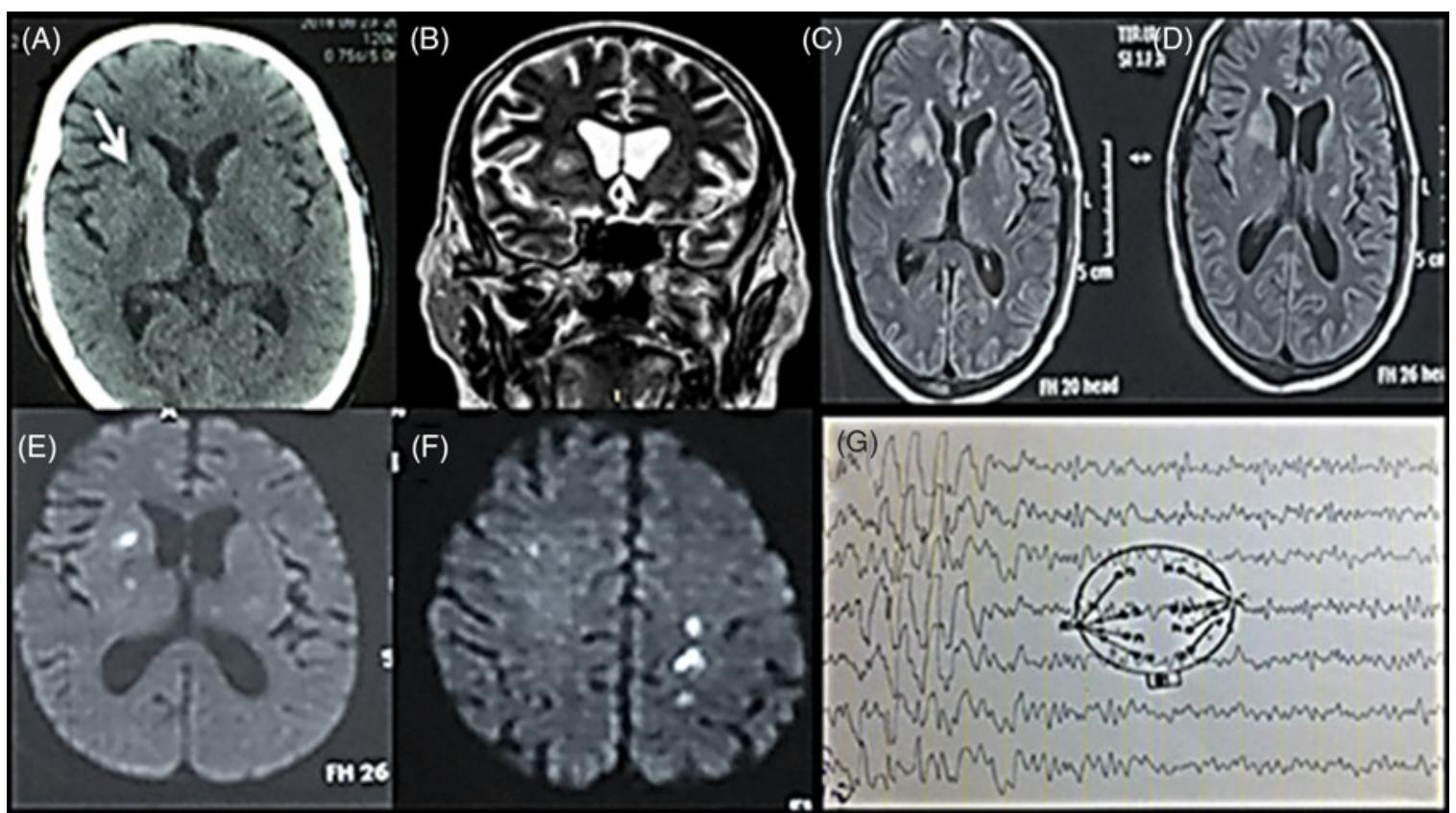

Figure 6: Neuroimaging and EEG of Case 5 with dystonia. CT-brain (A) showed hypodense lesion in the right putamen. MRIbrain showed hyperintense lesion in T2WI $(B), F L A I R(C$ and $D)$, and DWI (E and $F)$ in the right putamen and discrete bilateral subcortical WMHs. EEG showed generalized spike-wave complexes which were recorded in temporal relationship to myoclonic jerks $(G)$.

[urea: $12 \mathrm{mmol} / \mathrm{l}$; creatinine: $860 \mu \mathrm{mol} / \mathrm{l}$; RBS: $5.5 \mathrm{mmol} / \mathrm{l}$; albumin: $3.2 \mathrm{~g} / \mathrm{l}$; calcium: $1.875 \mathrm{mmol} / \mathrm{l}(7.5 \mathrm{mg} / \mathrm{dl})$; sodium: $124 \mathrm{mmol} / \mathrm{l}$; potassium: $3.4 \mathrm{mmol} / \mathrm{l}$; phosphorus: $4.5 \mathrm{mg} / \mathrm{dl}$; magnesium: $2.8 \mathrm{mg} / \mathrm{dl}$; HGB: $9.0 \mathrm{mg} / \mathrm{dl}$; RBCs count: $3.5 \mathrm{million} / \mathrm{mcl}$; $\mathrm{PH}$ : 7.32; PO2: $80 \mathrm{mmHg}$; PCO2: $44 \mathrm{mmHg}$; HCO3: $10 \mathrm{MEq} / \mathrm{l}$. She did not receive specific treatment for her condition. Neurologic evaluation (at the age of 60 , at the period of the study) showed presence of mixed movement disorder described as intermittent myoclonic movements of facial muscles, head, and trunk. At times, they looked synchronous in lower and upper face (perinasal contractions) with additional slower movements in the mouth as well as more prolonged jaw opening (dystonic). Some of the movements seemed to have a repetitive, semiregular (semiperiodic) manner. These episodes were frequent ( $\sim-10$ times per day particularly the day before dialysis). Interviewing of the patient when she was not encephalopathic revealed presence of infrequent facial and jaw movements (perinasal contractions and dystonic jaw movements). EEG showed generalized spike-wave complexes which were recorded in temporal relationship to the myoclonic jerking. Laboratory results showed high urea and creatinine, mild hypocalcemia, anemia, hyponatremia, and metabolic acidosis [urea: $11.8 \mathrm{mmol} / \mathrm{l}$; creatinine: $780 \mu \mathrm{mol} / \mathrm{l}$; RBS: $5.5 \mathrm{mmol} / \mathrm{l}$; albumin: $3.4 \mathrm{~g} / \mathrm{l}$; calcium: $8.7 \mathrm{mg} / \mathrm{dl} \quad(2.175 \mathrm{mmol} / \mathrm{l})$; sodium: $129 \mathrm{mmol} / \mathrm{l}$; potassium: $3.4 \mathrm{mmol} / \mathrm{l}$; phosphorus: $4.5 \mathrm{mg} / \mathrm{dl}$; magnesium: $2.8 \mathrm{mg} / \mathrm{dl}$; HGB: $8.9 \mathrm{mg} / \mathrm{dl}$; RBCs count: 3.2 million/mcl; PH: 7.34; PO2: 88 mmHg; PCO2: $40 \mathrm{mmHg}$; HCO3: $17 \mathrm{MEq} / \mathrm{l}$; thiamine: $85 \mathrm{nmol} / \mathrm{l}$; venous lactate: $0.60 \mathrm{mmol} / \mathrm{l}$ ]. MRI-brain showed hyperintense lesion in T2WI (B), FLAIR (C and D), and DWI (E and F) in the right putamen which was similar to CT findings (focal encephalomalacia) and discrete subcortical WMHs in both hemispheres.

\section{Discussion}

This cohort study prospectively followed a group of patients with uremia due to CKD over a period of 2 years for the presence of BG lesions and their related movement disorders, their risk variables, and neurologic outcomes. Selective BG lesions were observed in nearly one fifth of patients $(21.4 \%$ ), of them $75 \%$ developed movement disorders if we excluded cases with BG calcifications. To our knowledge, this is the largest cohort which followed patients with uremia without or with BG lesions and its related movement disorders for 2 years to determine their clinical and neuroimaging outcomes. In the literature, there are approximately 40 case reports of extrapyramidal movement disorders due to BG lesions with uremia ${ }^{4-23}$. Among them, only Wang and Cheng ${ }^{5}$ prospectively studied six patients with uremia who had BG-related movement disorders. The authors also retrospectively reviewed the clinical records of a large population of uremic patients in their institution and identified another six cases (Supplementary Table 2). In this study, we observed that patients with BG lesions whether they had movement disorders or not had characteristic risk variables which distinguished them from entire group and from those without BG lesions. The six patients with BG-related movement disorders developed uremia at a median age of 55 years (range: 50-58 years). They developed acute/ subacute onset of movement disorders after a median onset of uremia of 10 months (3-24 months). The majority (83\%) was females and had T2D $(66.6 \%)$ for a median duration of 8.6 years (range: 5-10). At the onset of movement disorders, higher renal chemistries than usual levels were found in $5(83.3 \%)$, metabolic acidosis was found in four (66.7\%) (Case 1, 2, 3, and 6) and hyponatremia was found in three (50\%) (Case 3, 4, and 6). None had thiamine deficiency or lactic acidosis. Review of the literature 
of case reports of patients with uremia and extrapyramidal movement disorders showed a median age at presentation of the movement disorder of 55 years (range 35-77 years). More than $90 \%$ of patients had diabetes for a median duration of 16 years (range: 5-23 years). High levels of urea and creatinine were observed in all patients (100\%), metabolic acidosis was observed in $90 \%$ of patients at presentation, and less than $20 \%$ had lactic acidosis (which was attributed to thiamine deficiency or metformin therapy). ${ }^{4-23}$

In this study, the classic triad of Parkinsonism (rest tremors, bradykinesia, and rigidity) was found in three patients $(75 \%)$ (Case 1, 4, and 5) but only bradykinesia was present in one (25\%) (Case 3), choreo-dystonia (generalized chorea and limb dystonia) was found in one (Case 2), and one had facial and jaw dystonia (Case 6). Previously reported cases with extrapyramidal movement disorders caused by uremia showed that the predominant clinical features included acute/subacute onset of symptoms with early-onset gait disorder and postural instability, symmetric bradykinesia or akinesia and rigidity, mild or absent rest tremors, and combination of other symptoms as dysarthria, dysphagia, or ataxia. $^{6-13}$ Typical features of Parkinsonism (rest tremors, bradykinesia, and rigidity) were reported in only $19 \%$ of patients with uremia. $^{4,13}$ There were also case reports of chorea ${ }^{14-22}$ and dystonia. $^{23}$

In our cases with Parkinsonism, the radiological aspects were bilateral (more or less symmetrical) lesions in the BG without (Case 1) or with involvement of the thalami (Case 3) or unilateral BG lesions (Case 4 and 5). These lesions had the following characteristics: (1) hypointense in T1WI and hyperintense in T2WI and FLAIR in right lentiform nucleus and left putamen with normal appearance of the ventricles and no mass effect (Case 1) suggesting metabolic, ischemic, hypoxia insults. However, the complete resolution of the lesions (seen in follow-up imaging) together with the patient's clinical circumstances at the onset suggests the possibility of reversible ischemic insult. (2) Heterogeneous intensity in T1WI (hypointense BG and left thalamic hyperintense lesions) and hyperintense in T2WI and FLAIR, hyperintense in the periphery (vasogenic edema) and hypointense in the center (cytotoxic edema) in ADC map and mass effect (Case 3). The lesions showed complete resolution in follow-up imaging. (3) Hyperintense lesion in ADC and FLAIR imaging in the right putamen and globus pallidus externa (cystic encephalomalacia) (Case 4). The presence of WMHs (small vessel disease) highly suggests that the focal encephalomalacia could be a consequence of ischemic insult. (4) Hypointense lesion in T1WI and ADC and hyperintense in T2WI and FLAIR in the right lentiform nucleus and no mass effect (Case 5). The presence of small hyperintense lesion in the right globus pallidus FLAIR and hypointense lesion in DWI (focal encephalomalacia) in follow-up indicate that the lesion was an ischemic insult.

In our cases with choreo-dystonia and dystonia, the radiological aspects were bilateral symmetrical lesions in the BG (Case 2) or unilateral BG lesions (Case 6). They had the following characteristics: (1) hypointense lesions in T1WI and hyperintense in T2WI, FLAIR, and DWI in putamen and globus pallidus externa bilaterally with normal appearance of the ventricles and no mass effect (Case 2) suggesting metabolic, ischemic, and/or hypoxia insults. However, the complete resolution of the lesions (seen in follow-up imaging) together with the patient's clinical circumstances at the onset suggests the possibility of reversible ischemic insult. (2) Hypodense lesion in the right putamen in CT which was hyperintense in T2WI, FLAIR, and DWI (Case 6). The presence of WMHs (small vessel disease) suggests that this lesion could be ischemic in nature.

Previous studies which used more sensitive MRI techniques as perfusion/diffusion and MRI spectroscopy described discrete or widespread BG lesions which were usually bilateral and symmetrical. Involvement of the surrounding white matter was observed in some patients. These lesions were collectively classified into two types $8,10,11,22,24,25$ : (a) hypointense in T1WI and hyperintense in T2WI, FLAIR, and DWI (in up to $88 \%$ of cases) indicating metabolic dysfunction (as hyperglycemia, hypoglycemia, hypoxia, and metabolic acidosis). The increased signal intensities with DWI indicated cytotoxic edema due to ischemia. ${ }^{8,10}$ High signal intensity in ADC indicated vasogenic edema. In some patients (20\%), ADC showed heterogeneous intensities such as low signal intensity indicating cytotoxic edema surrounded by high signal intensity of perifocal edema and consequent mass effect indicating vasogenic edema $^{10}$ or demyelination..$^{22,24}$ (b) Hyperintense in T1WI in association with cerebral parenchymal calcification due to hyperparathyroidism ${ }^{25}$ or in association with cerebral manganism states. ${ }^{11}$

It seems that the presented six cases with movement disorders had variable neurological and imaging outcomes. Persistence of the clinical manifestations was observed in three (Case 2, 5, and 6 ), progression was observed in two (Case 1 and 4) and regression of severe neurologic manifestations at onset was observed in one (Case 3). Complete resolution of neuroimaging was observed in three (Case 1, 2, and 3) but persistent imaging abnormalities were observed in three (Case 4, 5, and 6). Regarding the neurologic outcomes of previously reported cases, it has been shown that although there were shares and overlap in the demographic, clinical, and neuroimaging features, however, the prognoses or clinical outcomes of patients with movement disorders due to uremia were variable. For example, the majority had spontaneous reversibility or regression of neuroimaging lesions within weeks (approximately 3-4 weeks). However, neurological improvement lagged behind improvement of BG lesions (i.e. improvement in imaging did not always correspond with neurological and clinical improvement). The previously reported outcomes were as follows: $20 \%$ showed slow but complete resolution, $50 \%$ improved but had residual deficits, and $30 \%$ had no improvement. $^{5,12}$ In the large cohort study of Wang and Cheng ${ }^{5}$, the authors observed that the 12 patients with extrapyramidal movement disorders were diabetic and had acute-onset Parkinsonism or dyskinesias and various symptoms of consciousness disturbance, dysarthria, dysphagia, or ataxia. They all had symmetrical bilateral BG lesions and their laboratory tests were significant for high urea, creatinine, and metabolic acidosis. The authors followed up six patients for 3-8 weeks and observed various neurologic outcomes despite the resolution of BG lesions in MRI-brains. We observed that reversibility of neuroimaging findings and partial improvement of patients' neurologic conditions occurred with increasing the frequency of dialysis and correction of higher levels of urea and creatinine and associated metabolic disturbances but there were poor or no response to treatments for movement disorders (e.g. levodopa/carbidopa, haloperidol, etc.). Similar findings were reported in previous studies. ${ }^{8-10,17,18,22}$ 
It has been stated that the pathogenesis of BG lesions and their related movement disorders in patients with uremia has not been clearly defined. Based on this and other studies, multiple etiologies are suggested which include: (1) The accumulation of uremic toxins, blood brain barrier (BBB) injury, and the long-term metabolic insults associated with uremia may cause derangement of regional cerebral cellular metabolism or induce a functional disturbance in smooth muscle cells of BG blood vessels (cerebral vessel myogenic autoregulatory mechanisms). The latter may result in hypoperfusion, hypometabolism, hypoxia, impaired dopamine metabolism, and decrease in dopamine turnover. ${ }^{27-29}$ The presence of higher frequency of RLS particularly in patients with BG lesions compared to those without support the notion that accumulation of uremic toxins with increase susceptibility of injury of BG may result in dopamine deficiency. Pharmacological, neurophysiological, and functional neuroimaging studies suggested that dopamine deficiency and decreased striatal D2 receptor activity are causes of idiopathic RLS suggesting that similar mechanisms may contribute to RLS with uremic syndrome. ${ }^{30}$ Dopamine modulates the function of motor and sensory spinal cord neurons and decreased dopamine levels in nervous system contributing to symptoms of RLS. ${ }^{30}$ (2) Combined diabetes and uremia makes BG injuries more easily. ${ }^{13,19}$ This is supported by the finding of high frequency of WMHs in patients with BG lesions which is consistent with the presence of long-standing vascular risks for atherosclerosis or arteriosclerosis. It has been observed that in patients with diabetes, there are vascular autoregulatory dysfunction and marked reduction of glucose metabolism in BG (especially putamen). ${ }^{6}$ Also hyperglycemia can disrupt BBB and produces a global decrease in regional cerebral blood flow, intracellular acidosis, accumulation of extracellular glutamate, brain edema, and decreased activity of $\gamma$-amino-butyric acid. $^{29}$ (3) Low thiamine concentration due to malnutrition may aggravate BG injury with uremia ${ }^{4,15}$ (as evidenced by the finding that patients with low thiamine concentrations also had hypoalbuminemia indicating malnutrition). It has been suggested that due to blocking of the citric acid cycle, it is possible for low thiamine concentrations to result in cellular hypoxia of $\mathrm{BG}$, reduction of striatal dopamine levels, and extrapyramidal motor disorders. ${ }^{28}$ (4) Uremia and its metabolic complications may trigger or accelerate the appearance or the progressive BG degeneration.

\section{Conclusions}

BG lesions occur in approximately one-fifth of patients with uremia due to CKD. Extrapyramidal movement disorders were evident in $75 \%$ of patients with BG lesions. The results of this study indicate that (1) there is no clear threshold for the development of movement disorders in patients with uremia, and the long-term outcome was more dependent on each patient's comorbidities and the associated complications. (2) The possible pathogenic mechanisms underlying these movement disorders are multifactorial including toxic/metabolic/nutritional and ischemic/ microvascular. The combined presence of uremic toxins, metabolic disturbances, and low thiamine concentration are aggravating factors for permanent BG dysfunction in predisposed patients (e.g. small vessel disease due to diabetes, hypertension, etc.). (3) The discrepancy between radiological and clinical improvement supports the presence of permanent neuronal impairment inflicted during active disease which is not detectable with neuroimaging. (4) As there is no specific treatment for these movement disorders due to uremia, prevention should focus on optimizing management of uremia and its associated metabolic derangements and medical conditions.

\section{CONFLICT OF INTEREST}

The authors declare that they have no conflicts of interest.

\section{STATEMENT OF AUTHORSHIP}

SAH, KOM, SKA, and JGM did the clinical evaluation of the patients and collection of serum samples. AEL helped greatly in the interpretation of videos of patients with uremia and abnormal involuntary movement (expert opinion). AOM did the lab testing. EMM and HA interpreted the neuroimaging of patients. All authors participated in the design of the study, statistical analysis, and drafting the manuscript. All authors read and approved the final manuscript.

\section{SUPPLEMENTARY MATERIAL}

To view supplementary material for this article, please visit https://doi.org/10.1017/cjn.2020.29.

\section{REFERENCES}

1. Enomoto M, Inoue $\mathrm{Y}$, Namba $\mathrm{K}$, Munezawa T, Matsuura M. Clinical characteristics of restless legs syndrome in end-stage renal failure and idiopathic RLS patients. Mov Disord. 2008; 23(6):811-6.

2. Young RR, Shahani BT. Asterixis: one type of negative myoclonus. Adv Neurol. 1986;43:137-56.

3. Chadwick D, French AT. Uraemic myoclonus: an example of reticular reflex myoclonus? J Neurol Neurosurg Psychiatry. 1979; 42(1):52-5.

4. Wang HC, Brown P, Lees AJ. Acute movement disorders with bilateral basal ganglia lesions in uremia. Mov Disord. 1998; 13(6):952-7.

5. Wang HC, Cheng SJ. The syndrome of acute bilateral basal ganglia lesions in diabetic uremic patients. J Neurol. 2003;250(8): 948-55.

6. Wang HC, Hsu JL, Shen YY. Acute bilateral basal ganglia lesions in patients with diabetic uremia: an FDG-PET study. Clin Nucl Med. 2004;29(8):475-8.

7. Ohtake T, Negishi K, Okamoto $\mathrm{K}$, et al. Manganese-induced Parkinsonism in a patient undergoing maintenance hemodialysis. Am J Kidney Dis. 2005;46(4):749-53.

8. Lee PH, Shin DH, Kim JW, Song YS, Kim HS. Parkinsonism with basal ganglia lesions in a patient with uremia: evidence of vasogenic edema. Parkinsonism Relat Disord. 2006;12(2):93-6.

9. Cupidi C, Piccoli F, La Bella V. Acute reversible Parkinsonism in a diabetic-uremic patient. Clin Neurol Neurosurg. 2006; 108(6):601-3.

10. Kim TK, Seo SI, Kim JH, Lee NJ, Seol HY. Diffusion-weighted magnetic resonance imaging in the syndrome of acute bilateral basal ganglia lesions in diabetic uremia. Mov Disord. 2006; 21(8):1267-70.

11. da Silva CJ, da Rocha AJ, Jeronymo S, et al. A preliminary study revealing a new association in patients undergoing maintenance hemodialysis: manganism symptoms and T1 hyperintense changes in the basal ganglia. Am J Neuroradiol. 2007; 28(8):1474-9.

12. Li JY, Yong TY, Sebben R, Khoo E, Disney AP. Bilateral basal ganglia lesions in patients with end-stage diabetic nephropathy. Nephrology. 2008;13(1):68-72. 
13. Nishimura Y, Shibata K, Funaki T, Ito H, Ito E, Otsuka K. A case of subacute Parkinsonism presenting as bilateral basal ganglia legions by MRI in diabetic uremic syndrome. Rinsho Shinkeigaku. 2013;53(3):217-23.

14. Hung SC, Hung SH, Tarng DC, Yang WC, Chen TW, Huang TP. Thiamine deficiency and unexplained encephalopathy in hemodialysis and peritoneal dialysis patients. Am J Kidney Dis. 2001;38(5):941-7.

15. Hung SC, Hung SH, Tarng DC, Yang WC, Huang TP. Chorea induced by thiamine deficiency in hemodialysis patients. Am J Kidney Dis. 2001;37(2):427-30.

16. Lee EJ, Park JH, Ihn Y, Kim YJ, Lee SK, Park CS. Acute bilateral basal ganglia lesions in diabetic uraemia: diffusion-weighted MRI. Neuroradiology. 2007;49(12):1009-13.

17. Park JH, Kim HJ, Kim SM. Acute chorea with bilateral basal ganglia lesions in diabetic uremia. Can J Neurol Sci. 2007;34(2):248-50.

18. Kiryluk K, Khan F, Valeri A. Acute chorea and bilateral basal ganglia lesions in a haemodialysis patient. Kidney Int. 2008; 73(9):1087-91.

19. Shin YK, Hong SC, Ihn YK, Jeong JH, Han JH, Lee SP. A case of a patient with both chorea and restless legs syndrome. J Korean Med Sci. 2008;23(3):533-6.

20. Dicuonzo F, Di Fede R, Salvati A, et al. Acute extrapyramidal disorder with bilateral reversible basal ganglia lesions in a diabetic uremic patient: diffusion-weighted imaging and spectroscopy findings. J Neurol Sci. 2011;293(1-2):119.

21. Wali GM, Khanpet MS, Mali RV. Acute movement disorder with bilateral basal ganglia lesions in diabetic uremia. Ann Indian Acad Neurol. 2011;14(3):211-3.
22. Lin JJ. Generalized chorea in the syndrome of acute bilateral basal ganglia lesions in patients with diabetic uremia. J Clin Neurosci. 2011;18(9):1266-8.

23. Renard D, Castelnovo G, Taieb G, Verd A, Lionnet C, Labauge P. Bilateral basal ganglia lesions in a diabetic-uremic patient with dystonia. Acta Neurol Belg. 2011;111(2):166-7.

24. Tajima Y, Mito Y, Yanai M, Fukazawa Y. Unusual basal ganglia lesions in a diabetic uraemic patient proven to be demyelination: First pathological observation. BMJ Case Rep 2012:pii: bcr2012006522.

25. Grekas D, Balaskas E, Kampouris H, et al. Effective treatment of secondary hyperparathyroidism in hemodialysis patients by titration of intravenous calcitriol dosage. Clin Nephrol. 1999; 52(3):167-71.

26. Cogan MG, Covey CM, Arieff AI, et al. Central nervous system manifestations of hyperparathyroidism. Am J Med. 1978; 65(6):963-70.

27. Janavs JL, Aminoff MJ. Dystonia and chorea in acquired systemic disorders. J Neurol Neurosurg Psychiatry. 1998;65(4): 436-45.

28. Adachi N, Lei B, Deshpande G, et al. Uraemia suppresses central dopaminergic metabolism and impairs motor activity in rats. Intensive Care Med. 2011; 27(10):1655-60.

29. Kwok RP, Juorio AV. Concentration of striatal tyramine and dopamine metabolism in diabetic rats and effect of insulin administration. Neuroendocrinology. 1994;43(5):590-6.

30. Turjanski N, Lees AJ, Brooks DJ. Striatal dopaminergic function in restless legs syndrome: 18F-dopa and 11C-raclopride PET studies. Neurology. 1999;52(5):932-7. 